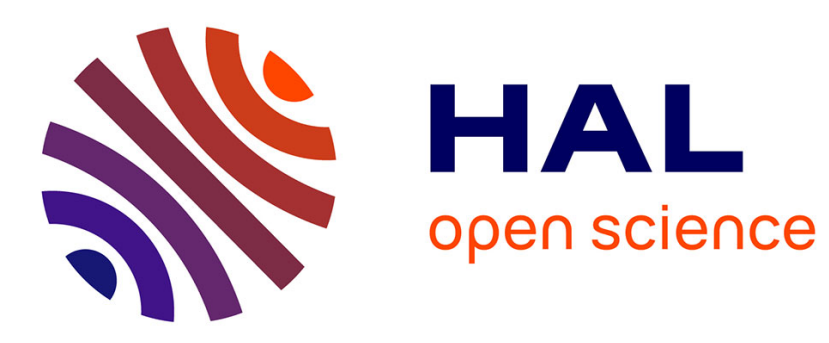

\title{
Cauchy Tetrahedron Argument applied to Higher Contact Interactions
}

F. Dell'Isola, A. Madeo, Pierre Seppecher

\section{To cite this version:}

F. Dell'Isola, A. Madeo, Pierre Seppecher. Cauchy Tetrahedron Argument applied to Higher Contact Interactions. Archive for Rational Mechanics and Analysis, 2016, 219 (3), pp.1305-1341. 10.1007/s00205-015-0922-6 . hal-01060548v2

\section{HAL Id: hal-01060548 \\ https://hal.science/hal-01060548v2}

Submitted on 14 Sep 2015

HAL is a multi-disciplinary open access archive for the deposit and dissemination of scientific research documents, whether they are published or not. The documents may come from teaching and research institutions in France or abroad, or from public or private research centers.
L'archive ouverte pluridisciplinaire HAL, est destinée au dépôt et à la diffusion de documents scientifiques de niveau recherche, publiés ou non, émanant des établissements d'enseignement et de recherche français ou étrangers, des laboratoires publics ou privés. 


\title{
Cauchy Tetrahedron Argument Applied to Higher Contact Interactions
}

\author{
F. Dell' Isola, A. Madeo, \& P. Seppecher
}

Communicated by I. FONSECA

\begin{abstract}
Second gradient theories are nowadays used in many studies in order to describe in detail some transition layers which may occur in micro-structured materials and in which physical properties are sharply varying. Sometimes higher order theories are also evoked. Up to now these models have not been based on a construction of stresses similar to the one due to Cauchy, which has been applied only for simple materials. It has been widely recognized that the fundamental assumption by Cauchy that the traction depends only on the normal of the dividing surface cannot be maintained for higher gradient theories. However, this observation did not urge any author, to our knowledge, to revisit the Cauchy construction in order to adapt it to a more general conceptual framework. This is what we do in this paper for a continuum of grade $N$ (also called $N$-th gradient continuum). Our construction is very similar to the one due to Cauchy; based on the tetrahedron argument, it does not introduce any argument of a different nature. In particular, we avoid invoking the principle of virtual work. As one should expect, the balance assumption and the regularity hypotheses have to be adapted to the new framework and tensorial computations become more complex.
\end{abstract}

\section{Contents}

1. Introduction

2. Notation

2.1. Measures

2.2. Tetrahedrons

2.3. Tensors

3. Cauchy Tetrahedron Argument

3.1. Representation Theorem for Surface Actions

3.2. Representation Theorem for Edge Actions

3.3. Representation Theorem for Wedge Actions

4. Extension to More General Shapes 
4.1. Domains and Shapes

4.2. Regularity Assumptions .

4.3. Generalization of Noll's Theorem .

5. An Algorithm for the Representation of Lower Order Contact Interactions

6. First, Second and Third Gradient Theories

6.1. Cauchy First Gradient Theory

6.2. Second Gradient Theory

6.3. Third Gradient Theory

7. Conclusions

\section{Introduction}

At the beginning of the 19th century A. L. CAUCHY founded Continuum Mechanics by assuming that two parts of a continuum interact by means of surface densities of contact forces concentrated on their dividing surface. Then, assuming that these contact forces depend only on the normal to the dividing surface and that they are balanced by some volume density of force (including inertia), he played with tetrahedrons and proved the existence of the so-called Cauchy stress tensor.

Many authors consider this point of view as the untouchable basis of Continuum Mechanics (see for example [27,50], and the criticism raised in [5] and in [15]). In 1959, W. NoLL [34] crystallized this faith by proving that the so-called Cauchy Postulate, the dependence of contact forces only on the normal of dividing surfaces, is indeed equivalent to the apparently weaker assumption of uniform boundedness of contact forces for all dividing surfaces. ${ }^{1}$ The merit of Noll's results consists in pointing out the relationship between the tetrahedron argument and measure theory (see for example [17]); the drawback is in camouflaging behind a technical hypothesis the physical assumption that the contact forces depend only on the normal.

Many other authors (see for example [1,4,6,9,20,23,26,33,39,41,43,48,51]) are accustomed to using second gradient theories to describe various phenomena in which strong variations of material properties occur. They thus use a theory based on the principle of virtual work and, at least since ToupIN [46,47], they are aware of the fact that the Cauchy Postulate is not valid in this context. Indeed, in [22], it is stated that "A central consequence of Toupin's work is the observation that Cauchy's hypothesis that the surface traction at a point $x$ on a surface $S$ depends on $S$ through its normal field at $x$ is not valid in a theory involving second gradients of the deformation, because in Toupin's theory the traction depends also on the curvature of $S$ at $x$ ". However, and remarkably enough, almost no effort (except, to our knowledge, the work [11]) has been made to adapt that Postulate and the tetrahedron construction to encompass theories of higher gradient continua.

All modern mechanicians admit that the introduction of surface contact interactions is the result of some underlying asymptotic process: from discrete atomic networks to continuum models, from microscopically non-homogeneous continua to

1 We note here that the fact of calling this hypothesis Postulate has been greatly misleading. Actually it is not a fundamental Principle of Mechanics as is sometimes believed, but a constitutive assumption. 
macroscopic homogenized effective ones, or from thin three-dimensional structures to their lower dimensional limits (that is shells, plates, beams). Contact mechanical interactions, that is, physical entities which act on the kinematics, need not necessarily be forces. They can be, for instance, couples. It is well known that a distribution of forces, through an asymptotic process, can be transformed into an interaction of a different type, for instance a couple. We remark that the need of considering more general contact interactions is universally accepted in the theory of beams, plates and shells, where the macro-model has a lower dimension when compared to the corresponding micro-model, whereas it has been regarded as controversial when considering continuum three-dimensional models whose microscopic counterparts are discrete systems of particles moving in a three-dimensional space or again three-dimensional continuum models.

The best way of describing a mechanical interaction, be it either a force or another type of more complex interaction, is to introduce its power expended on all admissible kinematic fields $U$. This is a linear form called virtual power. The fact of describing the interactions through their expended virtual power should not be confused with the so-called virtual power method. This method, popularized by GERMAIN [25] consists in making assumptions on the internal virtual power and deducing properties of contact interactions in a logical order which reverses the one used by CAUCHY [14]. It is remarkable that already GABRIO PIOLA, in his pioneering works $[16,37,38]$, raises several important theoretical questions about this subject. He understood, by means of a micro-macro identification procedure based on the Principle of Virtual Work, that, in general, one cannot assume that macroscopic contact interactions reduce simply to contact forces, and he introduced continuum models which are much more general than the one studied by CAUCHY. Such models have been considered for engineering applications only in the first decade of the 21 st century (see [15]).

Many results are now available (see for example [1,3,36,44,48] or [2]) indicating that it is physically needed and mathematically consistent to consider macroscopic continuum models where contact interactions arise which expend power on high order velocity gradients calculated on dividing surfaces (see also [13]). An essential common ingredient of many systems which, after a homogenization procedure, were found to present non-Cauchy contact interactions is that they show highly contrasted physical properties at the micro-level (see also [8,9]). On the purely macroscopic point of view, the necessity of considering such interactions has been proven in some elegant papers [24,25,28-30], when one wants to consider continuum models in which deformation energy may consistently depend on second or higher gradients of displacement. The conceptual framework introduced, for example, by TRUESDELL and NoLL [49], is not suitable for encompassing such models (see for example the difficulties arising in [18] and clarified in [11,14]). Moreover, the misunderstood range of validity of Noll's theorem persuaded many authors that the dependence of the deformation energy on higher gradients was forbidden by the second principle of thermodynamics (see for example [27] and [10]) or that the principles of thermodynamics needed to be modified $[18,32]$.

The present paper adapts the tetrahedron argument to include a class of continuum models which is much wider than the one originally considered by Cauchy. 
For our purposes it is needed to reconsider the assumptions on which Cauchy based his analysis. We explicitly discuss them here because (as is always true for any theory and model), in order to generalize Cauchy analysis, one has to have a clear understanding of its limits and to be aware of the fact that often its results are used outside the correct context. In his paper De la pression ou tension dans un corps solide, CAUCHY wrote ([7] p. 61 lines 3, 15) : "a small element experiences on its different faces and at each point of them a determined pressure or tension ... which can depend on the orientation of the surface. This being set, ...". Therefore he was clearly aware that he was accepting the two assumptions:

(H1) Contact interactions reduce to surface forces on the boundary.

(H2) Contact interactions depend only on the normal to the boundary.

Later he added (p. 63 lines 16): "Equilibrium should hold between inertialforce and the forces to which are reduced all pressures and tensions exerted on the surfaces, ...". Therefore he accepted the third assumption

Contact interactions are balanced by volume forces.

In his proof he applied the balance of forces to domains "with a very small volume, so that every dimension is an infinitesimal quantity of first order the mass being an infinitesimal quantity of the third order" (p. 62 line 9 and p. 64 line 4), and finally he stated that pressure and tension on a small face "experience, by moving from one point to another one on a face, infinitesimal variations of the first order" (p. 62 line 14). Therefore he implicitly accepted the regularity assumption:

(H4) Contact interactions depend continuously on the position.

The key conceptual advancement performed of the present paper consists of the acceptance of a wider class of contact interactions by weakening assumption (H1) [and this cannot be done without getting rid also of (H2)]. An attempt of weakening assumption ( $H 1)$ in order to model edge forces has been tried in [35] without introducing contact distributions of an order larger than one, yet it has been proven in [12] that both types of interactions are intrinsically linked. In a very relevant paper, SEGEV and DE BoTTON [42] have considered both the concentration of contact interactions on singular subsets of the boundary and higher order contact actions. They have proven, using assumptions which are different from ours, that a representation of the power of contact interactions in terms of hyperstresses actually exists. This representation is global and, contrary to Cauchy's approach, does not make explicit the relationship between hyperstress tensors and contact interactions, nor the way in which contact interactions depend on the shape of the boundary.

In this paper, we show, by following as closely as possible the spirit of Cauchy's works, how one can indeed consider continuum models in which contact distributions of order larger than one can arise or can concentrate on the geometrical singularities of the dividing surfaces (edges and wedges). We assume that, for a rich enough class of sub-bodies, the contact interactions exerted on a sub-body $B$ is a distribution concentrated on the dividing surface $\partial_{2} B$ and on its singularities 
of dimension one $\partial_{1} B$ and of dimension zero $\partial_{0} B{ }^{2}$ We also assume (and this is an actual restriction on the range of applicability of our theory) that the order of these distributions is uniformly bounded for all possible sub-bodies.

We take advantage of the fundamental theorem in the theory of distributions due to LAURENT SchWARTz, which states that every distribution having support included in a regular embedded sub-manifold $M$ can be uniquely decomposed as a finite sum of transverse derivatives of extensions of distributions defined on $\mathrm{M}$ (see [40])

$$
\sum_{k=0}^{N}<\mathrm{F}_{k}, \nabla_{\perp_{M}}^{k} U>_{M}
$$

At this point we need to formulate an assumption which may seem of a purely technical nature but which indeed has a limiting effect on the described phenomena. We decide to limit our attention to contact interactions for which the distributions $\mathrm{F}_{k}$ can be represented by smooth functions.

Note that, already in Cauchy framework where only surface 0-order distributions on surfaces are considered, a similar assumption is used to prove the Cauchy representation theorem. Our results, no more than those obtained by Cauchy, cannot encompass stress states for which there are stress concentrations along lower dimensional manifolds; models which are needed if one wants to model for instance a three dimensional elastic body containing a free moving two dimensional plate or a fluid containing some unknown interfaces endowed with surface tension. It is remarkable that there is no sound theoretical understanding of these phenomena which are, on the other hand, commonly studied and considered by physicists since the formulation of the Laplace and Young formula. Such a theoretical framework should include the capability of considering distributions on the surface which are measures but not functions. An interesting step in this direction has been performed in [4] when the localization of stress is a priori known. In [42], a way for attacking this problem is also given, but it is not the subject of the present paper.

Thus we write the power of contact interactions $\mathfrak{S}(B, U)$ by means of the representation ${ }^{3}$

$$
\mathfrak{S}(B, U)=\sum_{k=0}^{N-1} \int_{\partial_{2} B} \mathrm{~F}_{k}^{2}\left|\nabla_{\perp}^{k} U+\sum_{k=0}^{N-2} \int_{\partial_{1} B} \mathrm{~F}_{k}^{1}\right| \nabla_{\perp}^{k} U+\sum_{k=0}^{N-3} \int_{\partial_{0} B} \mathrm{~F}_{k}^{0} \mid \nabla_{\perp}^{k} U .
$$

The function $\mathfrak{S}$ characterizes the stress state of the continuum which is then said to be in a stress state of order $N$. The fields $\left(\mathrm{F}_{k}^{2}, \mathrm{~F}_{k}^{1}, \mathrm{~F}_{k}^{0}\right)$ are functions which depend on $B$ and on the position. They are dual quantities to the normal gradients $\nabla_{\perp}^{k} U$

\footnotetext{
${ }^{2}$ We do not post any difference here between the material sub-body and the subset it is occupying in the physical space. The regularity hypotheses we assume for such a subset will be precised later.

3 In the formula (1) the chosen summation bounds may seem restrictive. This is not the case, as one can easily add some extra terms with vanishing densities. We will see later on in this paper the reason for writing the distribution in this form.
} 
of the virtual velocity field and are called the contact $k+1$-forces. They are, by definition, orthogonal to the shape where they are applied. Thus $\mathrm{F}_{k}^{i}\left|\nabla_{\perp}^{k} U=\mathrm{F}_{k}^{i}\right|$ $\nabla^{k} U$ and in the sequel it will not be necessary to specify that only the orthogonal part of $\nabla^{k} U$ is involved.

The kinematics of considered continua may here be very general (for example the one specified in [19]). The configuration field may take values in a manifold and the velocity field in its tangent bundle, which can be of any tensorial nature. This tensorial nature is irrelevant in our developments and therefore, for the sake of efficiency, we operate as if the kinematics were described by a real valued function $U$. Therefore the tensor $\nabla_{\perp}^{k} U$ is considered to be of order $k$, as well as its dual quantities, and $F_{k}^{i} \mid \nabla_{\perp}^{k} U$ denotes the scalar product of the indicated tensors. It is straightforward, by applying our results component-wise, to extend them to the case where $U$ is a tensor, and in particular to the classical case where $U$ is a vector.

The class of sub-bodies that we consider must contain tetrahedrons if we want to follow the trail of Cauchy. Therefore, it cannot be limited to domains with smooth boundaries. In the first part of the paper we consider only tetrahedrons. Later on we extend the class of admissible sub-bodies to domains which are locally diffeormorph to tetrahedrons; their boundaries (which may be considered as Cauchy dividing surfaces) are piece-wise regular, with normal fields subjected to jumps on a finite set of regular curves eventually concurring into wedges.

One of the points of Cauchy the approach which is more often discussed (see for example [34] or [21]) is about the assumptions which are needed concerning the dependence of the fields $F_{k}^{i}$ upon the sub-body $B$. In this paper, we only suppose that this dependence is local. We also assume that the densities $F_{k}^{i}$ depend in a sufficiently regular way on the position when a sub-body is translated.

The theory of Cauchy is a particular case of the one that we present here; indeed, if we make the extra assumption that the stress state is of order one then we are back to the framework used by Cauchy and our demonstrations and results are identical.

Assuming that the stress state is of order one is indeed a constitutive assumption so deeply rooted in the mind of many authors that it has been very often accepted unconsciously and we emphasize that NoLL's theorem [34] cannot be proven without this assumption.

The generalized contact interactions we previously described are not usually considered in the literature. One can find two different reasons for this circumstance. First, the virtual work is not always the preferred tool for some mechanicians while it gives the conceptual framework in which generalized contact interactions arise naturally. Secondly, it is a fact that many materials cannot sustain stress states of order larger than one.

Cauchy's proof of the existence of a stress tensor is based on the equilibrium of contact forces with a force which is assumed to be diffuse in the volume. We also need a similar assumption. We assume that quasi-balance of power holds: for every virtual velocity field $U$, there exists a constant $K_{U}$ such that,

$$
|\mathfrak{S}(B, U)| \leqq K_{U}|B| .
$$

Here $|B|$ denotes the Lebesgue measure of $B$. 
When considering only rigid virtual velocity fields $U$, one reduces inequality (2) to the quasi-balance of forces which is a weak form of the equilibrium condition used by Cauchy. As remarked in [12], quasi-balance of forces is not sufficient to obtain a description of a stress state of order two or higher and one has to apply the quasi-balance of power to non-rigid velocity fields.

While inequality (2) could seem a very weak assumption, we emphasize that it rules out some possible stress states as for instance those occurring in continua including material surfaces or continua including interfaces with Laplace surface tension.

This paper is organized as follows. We first fix some notation in Section 2. Then in Section 3 we state representation results of the highest order terms of the stress state when the considered domain is a tetrahedron. We define the boundary operators which will be proven to describe how the shape of the boundary influences the contact interactions. Then, closely following Cauchy's argument, we prove the existence of a unique tensor representing through these boundary operators, the highest order actions which are applied on the faces, edges and wedges of the boundary of the domain. Due to the complexity of the considered interactions, the proof is much more technical than the original one by Cauchy. The technicalities come also from the fact that we need to use a non-orthogonal tetrahedron in order to represent not only the surface terms but also the edge and wedge terms. We extend these results to more general shapes in Section 4 by generalizing Noll's theorem. To that end, we define precisely in this latter section what we call the "shape" of a body. Then we prove that the highest order terms of the stress state depends on the shape of the domain only through the tangent tetrahedral shape. In Section 5 we show how, using integration by parts on the different elements of the boundary, the previous results can be used to obtain representations of all terms is the stress state. Section 6 illustrates these results. Indeed we show how the first, second or third gradient theories can easily be recovered. In Appendix we have gathered some very technical results which are needed for the establishment of the proofs in Section 3 but which can be skipped in a first reading.

\section{Notation}

\subsection{Measures}

When $M$ is a smooth embedded compact manifold in the Euclidean space, we denote $|M|$ as the Hausdorff measure of $M$ : if $M$ is a volume, a surface, a line or a discrete system of points $|M|$ denotes respectively its volume, area, length or cardinal number. Integrals over $M$ are integrals with respect to the corresponding Hausdorff measure without explicitly mentioning it.

\subsection{Tetrahedrons}

A tetrahedron is the central tool for proving the Cauchy representation theorem of contact interactions. This is still true in our case. Thus we fix here some useful notations for geometrical quantities associated to a tetrahedron. 
Definition 1. For any point $\hat{x}$, any unit independent vectors $n_{1}, n_{2}, n_{3}$ (with negative determinant), any unit vector $n_{0}$ satisfying for all $j \in\{1,2,3\}$

$$
n_{0} \cdot n_{j}<0
$$

and for any positive real number $h_{0}$ we consider the non degenerated tetrahedron defined by

$$
\Delta\left(\hat{x}, n_{1}, n_{2}, n_{3}, n_{0}, h_{0}\right):=\left\{x:(x-\hat{x}) \cdot n_{j}>0,(x-\hat{x}) \cdot n_{0}<h_{0}\right\} .
$$

For $i \in\{0,1,2,3\}$, we denote $\mathcal{F}_{i}$ as the face of the tetrahedron having for unit outward normals $n_{i}$. For $j \in\{1,2,3\}$, we adopt the following convention : $j+1$ denotes the index following $j$ in a circular permutation of $\{1,2,3\}$. We introduce the vectors $e_{j}:=\frac{n_{j+1} \times n_{j+2}}{\left\|n_{j+1} \times n_{j+2}\right\|}$ which are unit tangent vectors to three edges denoted respectively $\mathcal{L}_{j}$. The point $\hat{x}$ is the vertex of the tetrahedron where these three edges concur. On each edge $\mathcal{L}_{j}$, the unit vectors orthogonal to the line $\mathcal{L}_{j}$, tangent respectively to the faces $\mathcal{F}_{j+1}$ and $\mathcal{F}_{j-1}$ and external to them are $v_{j+1}^{j}:=e_{j} \times n_{j+1}$ and $v_{j-1}^{j}:=-e_{j} \times n_{j-1}$.

The height of the tetrahedron corresponding to the face $\mathcal{F}_{i}$ has length $h_{i}$. By projecting each edge vector $\left|\mathcal{L}_{j}\right| e_{j}$ onto the direction $n_{0}$ we get $\left|\mathcal{L}_{j}\right| e_{j} \cdot n_{0}=h_{0}$. By projecting $\left|\mathcal{L}_{j}\right| e_{j}$ onto the normal $n_{j}$ of $\mathcal{F}_{j}$, we obtain $h_{j}=-\left|\mathcal{L}_{j}\right| e_{j} \cdot n_{j}$. The volume of the tetrahedron is

$|\Delta|=\frac{1}{6} \operatorname{det}\left(\left|\mathcal{L}_{1}\right| e_{1},\left|\mathcal{L}_{2}\right| e_{2},\left|\mathcal{L}_{3}\right| e_{3}\right)=\frac{d h_{0}^{3}}{6\left(e_{1} \cdot n_{0}\right)\left(e_{2} \cdot n_{0}\right)\left(e_{3} \cdot n_{0}\right)}=\frac{1}{3}\left|\mathcal{F}_{i}\right| h_{i}$,

where $d:=\operatorname{det}\left(e_{1}, e_{2}, e_{3}\right)$. Thus we have, for $j \in\{1,2,3\}$,

$$
\left|\mathcal{F}_{j}\right|=-\left|\mathcal{F}_{0}\right| \frac{\left(e_{i} \cdot n_{0}\right)}{\left(e_{i} \cdot n_{i}\right)} .
$$

Finally, we remark that, for any $\bar{x}$ such that $(\bar{x}-\hat{x}) \cdot n_{0}=h_{0}$,

$$
\int_{\mathcal{L}_{j}}(x-\bar{x}) \cdot n_{0}=-\frac{\left|\mathcal{L}_{j}\right|^{2}}{2}\left(e_{j} \cdot n_{0}\right)=\frac{-h_{0}^{2}}{2\left(e_{j} \cdot n_{0}\right)}=-\frac{\left(e_{j+1} \cdot n_{0}\right)\left(e_{j+2} \cdot n_{0}\right)}{d}\left|\mathcal{F}_{0}\right| .
$$

\subsection{Tensors}

The Einstein convention of summation over repeated indices is used throughout this paper. The subscripts are relative to a three-dimensional basis.

Let $X$ and $Y$ be two tensors of order $p$ and $q$. We use the standard notation $\otimes$ for the tensorial product : $X \otimes Y$ is the $p+q$ tensor defined by

$$
(X \otimes Y)_{i_{1}, i_{2}, \ldots i_{p+q}}:=X_{i_{1}, i_{2}, \ldots i_{p}} Y_{i_{p+1}, \ldots i_{p+q}}
$$

while the product $\times$ stands for the cross product of two three dimensional vectors. We also use the notation $X^{\otimes r}$ for denoting the tensor of order $r \times p$ defined by 
induction by setting $X^{\otimes 1}:=X$ and $X^{\otimes r}=X^{\otimes r-1} \otimes X$. We use the standard notation $X \cdot Y$ for the contraction product : $X \cdot Y$ is the $p+q-2$ tensor defined by

$$
(X \cdot Y)_{i_{1}, i_{2}, \ldots i_{p+q-2}}:=X_{i_{1}, i_{2}, \ldots i_{p-1}, j} Y_{j, i_{p}, \ldots i_{p+q-2}} .
$$

If $p \geqq q$, we denote by $X \mid Y$ the $p-q$ tensor defined by

$$
(X \mid Y)_{i_{1}, i_{2}, \ldots, i_{p-q}}:=X_{i_{1}, i_{2}, \ldots i_{p-q}, j_{1}, j_{2}, \ldots, j_{q}} Y_{j_{1}, j_{2}, \ldots, j_{q}} .
$$

Note that, in the particular case $p=q$, this product coincides with the scalar product of tensors of order $p$, while, in the particular case $q=1$ (that is, when $Y$ is a vector), the product $X \mid Y$ coincides with $X \cdot Y$.

Given a permutation $\sigma$ in the symmetric group $\Sigma_{p}$ of permutations of $\{1, \ldots, p\}$, the tensor $\sigma X$ is defined by

$$
(\sigma X)_{i_{1}, i_{2}, \ldots, i_{p}}:=X_{i_{\sigma(1)}, i_{\sigma(2)}, \ldots, i_{\sigma(p)}} .
$$

We say that $X$ is completely symmetric if $\sigma X=X$ for every permutation $\sigma \in \Sigma_{p}$. The application which, to any tensor $X$ of order $p$, associates

$$
\mathcal{K}^{p}(X):=\frac{1}{p !} \sum_{\sigma \in \Sigma_{p}} \sigma X
$$

is the orthogonal projection onto the space of completely symmetric tensors; we call the completely symmetric part of $X$ its image under this projection. Note that, as the context always prevents any ambiguity, we omit the superscript $p$ in the sequel.

When $M$ is a smooth submanifold in the Euclidean space, we denote $\Pi_{M}$ as the orthogonal projector on the tangent subspace of $M$ and $\Lambda_{M}=I d-\Pi_{M}$ as the projector on its orthogonal subspace. For any tensor $X$ of order $p$ defined on $M$, we call completely orthogonal part of $X$ the tensor $\left(X_{\perp M}\right)_{i_{1} \ldots i_{p}}:=$ $X_{j_{1} \ldots . . j_{p}}\left(\Lambda_{M}\right)_{i_{1}}^{j_{1}} \ldots .\left(\Lambda_{M}\right)_{i_{p}}^{j_{p}}$ and we say that $X$ is completely orthogonal to $M$ if $X=X_{\perp M}$. We also denote $\mathcal{K}_{M}(X)$ the completely symmetric part of $X_{\perp M}$ :

$$
\mathcal{K}_{M}(X):=\mathcal{K}\left(X_{\perp M}\right)
$$

\section{Cauchy Tetrahedron Argument}

Let us consider a body submitted to a quasi balanced stress state $\mathfrak{S}$ of grade $N$; every tetrahedral sub-body $\Delta$ contained in this domain is submitted to a power of contact interactions $\mathfrak{S}(\Delta, U)$ given by

$$
\sum_{k=0}^{N-1} \int_{\partial_{2} \Delta} \mathrm{F}_{k}^{2}\left|\nabla_{\perp}^{k} U+\sum_{k=0}^{N-2} \int_{\partial_{1} \Delta} \mathrm{F}_{k}^{1}\right| \nabla_{\perp}^{k} U+\sum_{k=0}^{N-3} \int_{\partial_{0} \Delta} \mathrm{F}_{k}^{0} \mid \nabla_{\perp}^{k} U .
$$

We assume that quasi balance of power holds. The way the fields $\mathrm{F}_{k}^{q}$ depend on the considered sub-body will be studied in Section 4. However, this dependence 
is simple when a tetrahedron is concerned. As we require this dependence to be local we have to assume that (1) at any internal point of the four faces of the tetrahedron the surface actions $F_{k}^{2}$ depend only on the normal to the face; (2) at any internal point of its six edges the edge actions $F_{k}^{1}$ depend only on the dihedral shape characterized by the two normals concurring there and the tangent to the edge (for instance, using notation of Section 2.2 the dihedral shape $f_{1}$ at any internal point of $\mathcal{L}_{1}$ is characterized by $\left(n_{2}, n_{3}, e_{1}\right)$; (3) finally at any vertex of the tetrahedron the wedge actions $F_{k}^{0}$ depend only on the trihedral shape characterized by the triple of normals to the faces concurring in it [for instance the shape $w$ at $\hat{x}$ depends on $\left.\left(n_{1}, n_{2}, n_{3}\right)\right]$. In addition to this dependence upon the geometry of the considered sub-body, a dependence with respect to the position where the action is applied is also taken into account. We assume this dependence to be continuous.

We prove in this section, using the famous Cauchy tetrahedron argument, that the previous assumptions imply the existence of a unique completely symmetric tensor representing the highest order actions $\mathrm{F}_{N-1}^{2}, \mathrm{~F}_{N-2}^{1}$ and $\mathrm{F}_{N-3}^{0}$.

\subsection{Representation Theorem for Surface Actions}

Definition 2. At a regular point of a face $\mathcal{F}$ of a tetrahedron with external normal $n$, we define the boundary operator as $\mathcal{O}_{n}^{p}:=n^{\otimes(2 p-1)}$. It is a tensor of order $2 p-1$ characterized by the fact that, for any tensor $X$ of order $p$,

$$
\mathcal{O}_{n}^{p} \mid X:=X_{\perp \mathcal{F}} \cdot n \text {. }
$$

Lemma 1. At any point $\bar{x}$ and for any triplet of unit independent vectors $\left(n_{1}, n_{2}, n_{3}\right)$ (with negative determinant) there exists a continuous tensor field $\tilde{C}_{N}$ of order $N$ such that, for any unit vector $n_{0}$ satisfying the inequalities $n_{0} \cdot n_{j}<0$ for $j \in\{1,2,3\}$,

$$
\mathrm{F}_{N-1}^{2}\left(\bar{x}, n_{0}\right)=\mathcal{O}_{n_{0}}^{N} \mid \tilde{C}_{N}=\left(\tilde{C}_{N}(\bar{x}) \mid n_{0}^{\otimes N}\right) \otimes n_{0}^{\otimes N-1} .
$$

Proof. For any positive real number $\varepsilon$, we consider the one-parameter family of non degenerated homothetic tetrahedrons $\Delta^{\varepsilon}=\Delta\left(\bar{x}-\varepsilon n_{0}, n_{1}, n_{2}, n_{3}, n_{0}, \varepsilon\right)$ (cf. Definition 1) and we add a superscript $\varepsilon$ to all quantities associated to $\Delta^{\varepsilon}$ according to the notation stated in Section 2.2. We apply the quasi-balance of contact power for this family and for the fixed test field

$$
U: x \longrightarrow\left((x-\bar{x}) \cdot n_{0}\right)^{N-1} U_{0}
$$

where $U_{0}$ is generic in the space $\mathcal{E}$ which describes the kinematics of the continuum. We recall that, everywhere in the sequel, the tensorial nature of $U_{0}$ is overlooked. Without loss of generality $U_{0}$ is considered as a scalar quantity. Quasi-balance $\left|\mathfrak{S}\left(\Delta^{\varepsilon}, U\right)\right| \leqq K_{U}\left|\Delta^{\varepsilon}\right|$ reads

$$
\left|\sum_{k=0}^{N-1} \int_{\partial_{2} \Delta^{\varepsilon}} \mathrm{F}_{k}^{2}\right| \nabla^{k} U+\sum_{k=0}^{N-2} \int_{\partial_{1} \Delta^{\varepsilon}} \mathrm{F}_{k}^{1}\left|\nabla^{k} U+\sum_{k=0}^{N-3} \int_{\partial_{0} \Delta^{\varepsilon}} \mathrm{F}_{k}^{0}\right| \nabla^{k} U\left|\leqq K_{U}\right| \Delta^{\varepsilon} \mid .
$$


In the sequel we draw conclusions from the fact that $\left|\mathcal{F}_{0}^{\varepsilon}\right|^{-1}\left|\mathfrak{S}\left(\Delta^{\varepsilon}, U\right)\right|$ has a vanishing limit when $\varepsilon$ tends to zero. We start by remarking that the field $U$ together with all its derivatives up to order $N-2$ vanish on the plane $(x-\bar{x}) \cdot n_{0}=0$ which includes the face $\mathcal{F}_{0}^{\varepsilon}$ and the edges and wedges which border it. Indeed:

$$
\nabla^{k} U=\frac{(N-1) !}{(N-1-k) !}\left((x-\bar{x}) \cdot n_{0}\right)^{N-1-k} U_{0} \otimes n_{0}^{\otimes k} .
$$

This last equation also shows that, when $\varepsilon$ tends to zero, the asymptotic order of magnitude of $\nabla^{k} U$ in $\Delta^{\varepsilon}$ is $O\left(\varepsilon^{N-1-k}\right)$. Therefore, recalling the assumption according to which the stress state depends regularly on the space variables (18), (19), we get the estimates

$$
\begin{aligned}
& \left|\int_{\partial_{2} \Delta^{\varepsilon}} F_{k}^{2}\right| \nabla^{k} U|=| \partial_{2} \Delta^{\varepsilon} \mid O\left(\varepsilon^{N-1-k}\right)=O\left(\varepsilon^{N+1-k}\right), \\
& \left|\int_{\partial_{1} \Delta^{\varepsilon}} F_{k}^{1}\right| \nabla^{k} U|=| \partial_{1} \Delta^{\varepsilon} \mid O\left(\varepsilon^{N-1-k}\right)=O\left(\varepsilon^{N-k}\right), \\
& \left|\int_{\partial_{0} \Delta^{\varepsilon}} F_{k}^{0}\right| \nabla^{k} U \mid=O\left(\varepsilon^{N-1-k}\right)
\end{aligned}
$$

Thus, keeping only those terms which are not plainly vanishing in the limit of $\left|\mathcal{F}_{0}^{\varepsilon}\right|^{-1}\left|\mathfrak{S}\left(\Delta^{\varepsilon}, U\right)\right|$, we get

$$
\begin{aligned}
\lim _{\varepsilon \rightarrow 0}\left|\mathcal{F}_{0}^{\varepsilon}\right|^{-1} & \left(\sum_{i=0}^{3} \int_{\mathcal{F}_{i}^{\varepsilon}}(N-1) ! \mathrm{F}_{N-1}^{2} \mid\left(U_{0} \otimes n_{0}^{\otimes N-1}\right)\right. \\
& +\sum_{j=1}^{3} \int_{L_{j}^{\varepsilon}}(N-1) !\left((x-\bar{x}) \cdot n_{0}\right) \mathrm{F}_{N-2}^{1} \mid\left(U_{0} \otimes n_{0}^{\otimes N-2}\right) \\
& \left.+\frac{(N-1) ! \varepsilon^{2}}{2} \mathrm{~F}_{N-3}^{0}\left(\bar{x}-\varepsilon n_{0}, w\right) \mid\left(U_{0} \otimes n_{0}^{\otimes N-3}\right)\right)=0 .
\end{aligned}
$$

Making explicit the arguments of the functions $\mathrm{F}$ and applying the mean value theorem, we get

$$
\begin{aligned}
\lim _{\varepsilon \rightarrow 0}\left|\mathcal{F}_{0}^{\varepsilon}\right|^{-1} & \left(\sum_{i=0}^{3}\left|\mathcal{F}_{i}^{\varepsilon}\right| \mathrm{F}_{N-1}^{2}\left(x_{i}^{\varepsilon}, n_{i}\right) \mid\left(U_{0} \otimes n_{0}^{\otimes N-1}\right)\right. \\
& +\sum_{j=1}^{3}\left(\mathrm{~F}_{N-2}^{1}\left(\tilde{x}_{j}^{\varepsilon}, f_{j}\right) \mid\left(U_{0} \otimes n_{0}^{\otimes N-2}\right) \int_{\mathcal{L}_{j}^{\varepsilon}}\left((x-\bar{x}) \cdot n_{0}\right)\right) \\
& \left.+\frac{\varepsilon^{2}}{2} \mathrm{~F}_{N-3}^{0}\left(\bar{x}-\varepsilon n_{0}, w\right) \mid\left(U_{0} \otimes n_{0}^{\otimes N-3}\right)\right)=0
\end{aligned}
$$

for some points $x_{i}^{\varepsilon} \in \mathcal{F}_{i}^{\varepsilon}$ and $\tilde{x}_{j}^{\varepsilon} \in \mathcal{L}_{j}^{\varepsilon}$. Using the geometrical identities we have established in Section 2.2, we obtain 


$$
\begin{aligned}
& \lim _{\varepsilon \rightarrow 0}\left(\mathrm{~F}_{N-1}^{2}\left(x^{\varepsilon}, n_{0}\right)\left|\left(U_{0} \otimes n_{0}^{\otimes N-1}\right)-\sum_{j=1}^{3} \frac{\left(e_{j} \cdot n_{0}\right)}{\left(e_{j} \cdot n_{j}\right)} \mathrm{F}_{N-1}^{2}\left(x_{j}^{\varepsilon}, n_{j}\right)\right|\left(U_{0} \otimes n_{0}^{\otimes N-1}\right)\right. \\
& \quad+\sum_{j=1}^{3}\left(-d^{-1}\right) \frac{\left(e_{1} \cdot n_{0}\right)\left(e_{2} \cdot n\right)\left(e_{3} \cdot n\right)}{\left(e_{j} \cdot n_{0}\right)} \mathrm{F}_{N-2}^{1}\left(\tilde{x}_{j}^{\varepsilon}, f_{j}\right) \mid\left(U_{0} \otimes n_{0}^{\otimes N-2}\right) \\
& \left.\quad+d^{-1}\left(e_{1} \cdot n_{0}\right)\left(e_{2} \cdot n_{0}\right)\left(e_{3} \cdot n_{0}\right) \mathrm{F}_{N-3}^{0}\left(\bar{x}-\varepsilon n_{0}, w\right) \mid\left(U_{0} \otimes n_{0}^{\otimes N-3}\right)\right)=0 .
\end{aligned}
$$

Passing to the limit is now easy. Further using the arbitrariness of $U_{0}$, and introducing the second order tensors $E_{i}:=e_{i+1} \otimes e_{i+2}$, we get

$$
\begin{aligned}
\mathrm{F}_{N-1}^{2}\left(\bar{x}, n_{0}\right) \mid & n_{0}^{\otimes N-1}-\sum_{j=1}^{3} \frac{\left(e_{j} \cdot n_{0}\right)}{\left(e_{j} \cdot n_{j}\right)} \mathrm{F}_{N-1}^{2}\left(\bar{x}, n_{j}\right) \mid n_{0}^{\otimes N-1} \\
& -\sum_{j=1}^{3}\left(d^{-1}\right)\left(E_{j} \cdot\left(n_{0} \otimes n_{0}\right)\right) \mathrm{F}_{N-2}^{1}\left(\bar{x}, f_{j}\right) \mid n^{\otimes N-2} \\
& +d^{-1}\left(e_{2} \cdot n_{0}\right)\left(e_{1} \cdot n_{0}\right)\left(e_{3} \cdot n_{0}\right) \mathrm{F}_{N-3}^{0}(\bar{x}, w) \mid n_{0}^{\otimes N-3}=0,
\end{aligned}
$$

or equivalently,

$$
\begin{aligned}
\mathrm{F}_{N-1}^{2}\left(\bar{x}, n_{0}\right) \mid & n_{0}^{\otimes N-1}-\sum_{j=1}^{3} \frac{1}{\left(e_{j} \cdot n_{j}\right)}\left(\mathrm{F}_{N-1}^{2}\left(\bar{x}, n_{j}\right) \otimes e_{j}\right) \mid n_{0}^{\otimes N} \\
& -\sum_{j=1}^{3}\left(d^{-1}\right)\left(\mathrm{F}_{N-2}^{1}\left(\bar{x}, f_{j}\right) \otimes E_{j}\right) \mid n_{0}^{\otimes N} \\
& +d^{-1}\left(\mathrm{~F}_{N-3}^{0}(\bar{x}, w) \otimes e_{1} \otimes e_{2} \otimes e_{3}\right) \mid n_{0}^{\otimes N}=0
\end{aligned}
$$

Thus, defining the tensor field $\tilde{C}_{N}(\bar{x})$ by

$$
\begin{aligned}
\tilde{C}_{N}(\bar{x}):= & \sum_{j=1}^{3} \frac{1}{\left(e_{j} \cdot n_{j}\right)}\left(\mathrm{F}_{N-1}^{2}\left(\bar{x}, n_{j}\right) \otimes e_{j}\right)+\sum_{j=1}^{3} d^{-1}\left(\mathrm{~F}_{N-2}^{1}\left(\bar{x}, f_{j}\right) \otimes E_{j}\right) \\
& -d^{-1}\left(\mathrm{~F}_{N-3}^{0}(\bar{x}, w) \otimes e_{1} \otimes e_{2} \otimes e_{3}\right),
\end{aligned}
$$

we obtain $\mathrm{F}_{N-1}^{2}\left(\bar{x}, n_{0}\right)\left|n_{0}^{\otimes N-1}=\tilde{C}_{N}(\bar{x})\right| n_{0}^{\otimes N}$. As $\mathrm{F}_{N-1}^{2}\left(\bar{x}, n_{0}\right)$ is completely orthogonal to $\mathcal{F}_{0}^{\varepsilon}$, the previous equation implies

$$
\mathrm{F}_{N-1}^{2}\left(\bar{x}, n_{0}\right)=\left(\tilde{C}_{N}(\bar{x}) \mid n_{0}^{\otimes N}\right) \otimes n_{0}^{\otimes N-1},
$$

which concludes the proof.

Let us remark that formula (6) is a partial representation result. Indeed, as $\tilde{C}_{N}$ depends on $\bar{x}, e_{1}, e_{2}, e_{3}, n_{1}, n_{2}, n_{3}$ (that is on $\bar{x}, n_{1}, n_{2}, n_{3}$ ) but does not depend on 
$n_{0}$, this formula describes the way $\mathrm{F}_{N-1}^{2}(\bar{x}, n)$ depends on $n_{0}$. However, it is not valid for any unit vector $n_{0}$ in the sphere $S_{2}$ but only for those which belong to the cone defined by inequalities $n_{0} \cdot n_{i}<0 ; i \in\{1,2,3\}$. This is a common feature of all classical proofs based on the Cauchy tetrahedron argument, and the results are generally extended by using an action-reaction argument. Instead, we will use the purely topological Lemma 3 which states that an operator which, locally on the sphere, coincides with multilinear completely symmetric operators, coincides globally on the sphere with a completely symmetric operator. ${ }^{4}$ Let us begin by remarking that a multilinear completely symmetric operator is entirely defined when defined on some open subset of the sphere.

Lemma 2. Assume that two completely symmetric $p$-tensors $\mathcal{C}$ and $\mathcal{C}^{\prime}$ satisfy

$$
\mathcal{C}\left|n^{\otimes p}=\mathcal{C}^{\prime}\right| n^{\otimes p}
$$

for any $n$ in some open subset $O$ of the unit sphere $S_{2}$. Then $\mathcal{C}=\mathcal{C}^{\prime}$.

Proof. The completely symmetric $p$-tensor $\mathcal{C}^{\prime \prime}:=\mathcal{C}-\mathcal{C}^{\prime}$ satisfies $\mathcal{C}^{\prime \prime} \mid n^{\otimes p}=0$ for all $n \in O$ and therefore for all $n$ in the cone $D:=\{r n ; r \in \mathbb{R}, n \in O\}$. We use the polarization formula valid for any completely symmetric $p$-tensor (see [45] and references there cited):

$$
\begin{aligned}
\mathcal{C}^{\prime \prime} \mid\left(u_{1} \otimes u_{2} \otimes \cdots \otimes u_{p}\right) & =\frac{1}{2^{p} p !} \sum_{\xi \in\{-1,1\}^{p}} \xi_{1} \xi_{2} \cdots \xi_{p} \mathcal{C}^{\prime \prime} \mid\left(\sum_{i=1}^{p} \xi_{i} u_{i}\right)^{\otimes p} \\
& =\frac{1}{2^{p} p !} \sum_{\xi \in\{-1,1\}^{p}}\left(\xi_{p}\right)^{2 p} \frac{\xi_{1}}{\xi_{p}} \cdots \frac{\xi_{p}}{\xi_{p}} \mathcal{C}^{\prime \prime} \mid\left(\sum_{i=1}^{p} \frac{\xi_{i}}{\xi_{p}} u_{i}\right)^{\otimes p} \\
& =\frac{1}{2^{p-1} p !} \sum_{\xi \in\{-1,1\}^{p-1}} \tilde{\xi}_{1} \cdots \tilde{\xi}_{p-1} \mathcal{C}^{\prime \prime} \mid\left(\sum_{i=1}^{p-1} \tilde{\xi}_{i} u_{i}+u_{p}\right)^{\otimes p} .
\end{aligned}
$$

Let $y \in O$ and $r>0$ such that $B(y, p r) \subset D$ and let us consider the open subset $O^{\prime}$ of $\left(\mathbb{R}^{3}\right)^{p}$ defined by $O^{\prime}:=(0,0, \ldots, 0, y)+B(0, r)^{p}$. For any $\xi \in\{-1,1\}^{p-1}$ and any $\left(u_{1}, u_{2}, \ldots, u_{p}\right) \in O^{\prime}$

$$
\sum_{i=1}^{p-1} \xi_{i} u_{i}+u_{p} \in D
$$

As a consequence

$$
\mathcal{C}^{\prime \prime} \mid\left(\sum_{i=1}^{p-1} \xi_{i} u_{i}+u_{p}\right)^{\otimes p}=0
$$

\footnotetext{
4 It is remarkable that in the particular case of linear operators, Lemma 3 allows us to skip the classical Cauchy Action-Reaction lemma. Indeed, as soon as the Cauchy representation is proven to be valid in any trihedron, the linearity of $F_{2}^{2}(n)$ with respect to $n$ is assured on the whole sphere.
} 
and from the polarization formula we get $\mathcal{C}^{\prime \prime} \mid\left(u_{1} \otimes u_{2} \otimes \cdots \otimes u_{p}\right)=0$. Then the multilinear application $\mathcal{C}^{\prime \prime}$ vanishes in the open set $O^{\prime}$ and therefore is identically null.

Lemma 3. Let $\varphi$ be a function defined on the unit sphere $S_{2}$. Assume that, for any $\bar{n} \in S_{2}$, there exists an open neighborhood $O_{\bar{n}}$ of $\bar{n}$ in $S_{2}$ and a completely symmetric $p$-tensor $\mathcal{C}_{\bar{n}}$, such that, for any $n \in O_{\bar{n}}$,

$$
\varphi(n)=\mathcal{C}_{\bar{n}} \mid n^{\otimes p} .
$$

Then there exists a unique completely symmetric $p$-tensor $\mathcal{C}$ such that, for any $n \in S_{2}$,

$$
\varphi(n)=\mathcal{C} \mid n^{\otimes p} .
$$

Moreover, all tensors $\mathcal{C}_{\bar{n}}$ coincide with $\mathcal{C}$.

Proof. Let us fix $\bar{n} \in S_{2}$ and consider the largest open subset $O$ of $S_{2}$ such that $\varphi(n)=\mathcal{C}_{\bar{n}} \mid n^{\otimes p}$ for all $n \in O$. Clearly $O$ is a non-empty subset of $S_{2}$ as it contains $O_{\bar{n}}$. Let $\tilde{n} \in S_{2}$ belong to the closure of $O$. On the non-empty open set $O_{\tilde{n}} \cap O$, we have $\varphi(n)=\mathcal{C}_{\bar{n}}\left|n^{\otimes p}=\mathcal{C}_{\tilde{n}}\right| n^{\otimes p}$. From the previous lemma we know that $\mathcal{C}_{\bar{n}}=\mathcal{C}_{\tilde{n}}$ and so $\tilde{n} \in O$. The set $O$ is closed. The connectedness of $S_{2}$ implies that $O=S_{2}$, which concludes the proof.

The previous lemmas enable us to state the first representation theorem:

Theorem 1. There exists a unique continuous completely symmetric tensor field $C_{N}$ of order $N$ such that, at any point $\bar{x}$ and for any unit vector $n$,

$$
\mathrm{F}_{N-1}^{2}(\bar{x}, n)=\mathcal{O}_{n}^{N} \mid C_{N}=\left(C_{N}(\bar{x}) \mid n^{\otimes N}\right) \otimes n^{\otimes N-1} .
$$

Proof. Let us apply Lemma 1 for $\bar{x}$ and any triplet $\left(n_{1}, n_{2}, n_{3}\right)$ with negative determinant. As $n_{0}^{\otimes N}$ is a completely symmetric tensor, it is clear that Equation (6) remains valid if we replace $\tilde{C}_{N}$ by its completely symmetric part $C_{N}$. Note that the way we constructed $C_{N}(\bar{x})$ shows that this tensor depends continuously on $\bar{x}$. We get representation formulas for $\mathrm{F}_{N-1}^{2}(\bar{x}, n)$ valid in the open cones defined by inequalities (3). Then Lemma 3 establishes that this representation actually holds on the whole sphere $S_{2}$. It also establishes that, despite the way we defined it, the tensor $C_{N}$ does not depend on the triplet $\left(n_{1}, n_{2}, n_{3}\right)$. Moreover the uniqueness of the representation is assured.

\subsection{Representation Theorem for Edge Actions}

The dependence of the line actions upon the shape of the edge is more intricate. In order to define the appropriated boundary operator, we must first fix some notation. For any manifold $M$ and $1 \leqq q \leqq p$, we introduce the tensors $\mathcal{P}_{M, q}^{p}$ and 
$\mathcal{P}_{M}^{p}$ of order $2 p$ by setting, ${ }^{5}$ for any tensors $X, v, Y$ of order respectively $q-1,1$ and $p-q$,

$$
\begin{aligned}
\mathcal{P}_{M, q}^{p} \mid(X \otimes v \otimes Y) & :=X \otimes Y_{\perp M} \otimes\left(\Pi_{M} \cdot v\right) . \\
\mathcal{P}_{M}^{p} & :=\sum_{q=1}^{p} \mathcal{P}_{M, q}^{p} .
\end{aligned}
$$

Definition 3. We denote $\left[\mathcal{L}_{j}\right]$ as the pair of subscripts of the faces concurring on the edge $\mathcal{L}_{j}$. The shape $f_{j}$ of $\mathcal{L}_{j}$ is determined by the vectors $e_{j}, n_{k}, v_{k}^{j}\left(k \in\left[\mathcal{L}_{j}\right]\right)$. At a regular point of $\mathcal{L}_{j}$, the boundary operator $\mathcal{O}_{f_{j}}^{p}$ is the tensor of order $2 p-2$ defined by setting, for any tensor $X$ of order $p$,

$$
\mathcal{O}_{f_{j}}^{p} \mid X:=\sum_{k \in\left[\mathcal{L}_{j}\right]}\left(\mathcal{P}_{\mathcal{F}_{k}}^{p-1} \mid\left(X \cdot n_{k}\right)\right) \cdot v_{k}^{j} .
$$

Lemma 4. The tensor $\tilde{C}_{N}$ introduced by Lemma 2 also satisfies

$$
\mathrm{F}_{N-2}^{1}\left(\bar{x}, f_{1}\right)=\mathcal{K}_{\mathcal{L}_{1}}\left(\mathcal{O}_{f_{1}}^{N} \mid \tilde{C}_{N}\right)
$$

where, following the notation fixed in Section 2.2, $f_{1}$ denotes the shape of the edge $\mathcal{L}_{1}$ of a tetrahedron $\Delta\left(\hat{x}, n_{1}, n_{2}, n_{3}, n_{0}, h_{0}\right)$.

Proof. Let us now compute $\mathcal{K}_{f_{1}}\left(\mathcal{O}_{f_{1}}^{N} \mid\left(\tilde{C}_{N}\right)\right)$ by considering the different terms of formula (8). The term

$$
\begin{aligned}
\mathcal{O}_{f_{1}}^{N} \mid\left(\mathrm{F}_{N-1}^{2}\left(\bar{x}, n_{j}\right) \otimes e_{j}\right) & =\sum_{k \neq 1}\left(\mathcal{P}_{\mathcal{F}_{k}}^{N-1} \mid\left(\left(\mathrm{F}_{N-1}^{2}\left(\bar{x}, n_{j}\right) \otimes e_{j}\right) \cdot n_{k}\right)\right) \cdot v_{k}^{1} \\
& =\sum_{k \neq 1}\left(e_{j} \cdot n_{k}\right)\left(\mathcal{P}_{\mathcal{F}_{k}}^{N-1} \mid\left(\mathrm{F}_{N-1}^{2}\left(\bar{x}, n_{j}\right)\right) \cdot v_{k}^{1}\right.
\end{aligned}
$$

vanishes as $\left(e_{j} \cdot n_{k}\right)=0$ if $k \neq j$ and $\mathcal{P}_{\mathcal{F}_{k}}^{N-1} \mid\left(\mathrm{F}_{N-1}^{2}\left(\bar{x}, n_{j}\right)\right)=0$ if $k=j$. We have also, using the fact that $\left(e_{2}\right)_{\perp \mathcal{F}_{3}}=0$,

$$
\begin{aligned}
\mathcal{O}_{f_{1}}^{N} \mid & \left(\mathrm{F}_{N-3}^{0}(\bar{x}, w) \otimes e_{1} \otimes e_{2} \otimes e_{3}\right) \\
& =\sum_{k \neq 1}\left(\mathcal{P}_{\mathcal{F}_{k}}^{N-1} \mid\left(\left(\mathrm{F}_{N-3}^{0}(\bar{x}, w) \otimes e_{1} \otimes e_{2} \otimes e_{3}\right) \cdot n_{k}\right) \cdot v_{k}^{1}\right) \\
& =\left(e_{3} \cdot n_{3}\right)\left(\mathcal{P}_{\mathcal{F}_{3}}^{N-1} \mid\left(\mathrm{F}_{N-3}^{0}(\bar{x}, w) \otimes e_{1} \otimes e_{2}\right)\right) \cdot v_{3}^{1} \\
& =\left(e_{3} \cdot n_{3}\right) \sum_{q=1}^{N-1}\left(\mathcal{P}_{\mathcal{F}_{3}, q}^{N-1} \mid\left(\mathrm{F}_{N-3}^{0}(\bar{x}, w) \otimes e_{1} \otimes e_{2}\right)\right) \cdot v_{3}^{1}
\end{aligned}
$$

\footnotetext{
${ }^{5}$ Note that this formula simply reads $\mathcal{P}_{M, p}^{p} \mid(X \otimes v):=X \otimes\left(\Pi_{M} \cdot v\right)$ when $q=p$.
} 


$$
\begin{aligned}
& =\left(e_{3} \cdot n_{3}\right)\left(\sum_{q=1}^{N-2}\left(\mathcal{P}_{\mathcal{F}_{3}, q}^{N-1} \mid\left(\mathrm{F}_{N-3}^{0}(\bar{x}, w) \otimes e_{1} \otimes e_{2}\right)\right) \cdot v_{3}^{1}\right. \\
& \left.+\left(\mathcal{P}_{\mathcal{F}_{3}, N-1}^{N-1} \mid\left(\mathrm{F}_{N-3}^{0}(\bar{x}, w) \otimes e_{1} \otimes e_{2}\right)\right) \cdot v_{3}^{1}\right) \\
& =\left(e_{3} \cdot n_{3}\right)\left(\sum_{q=1}^{N-2}\left(\mathcal{P}_{\mathcal{F}_{3}, q}^{N-2} \mid\left(\mathrm{F}_{N-3}^{0}(\bar{x}, w) \otimes e_{1}\right)\right) \cdot v_{3}^{1} \otimes\left(e_{2}\right)_{\perp \mathcal{F}_{3}}\right. \\
& \left.+\left(\mathrm{F}_{N-3}^{0}(\bar{x}, w) \otimes e_{1} \otimes e_{2}\right) \cdot v_{3}^{1}\right) \\
& =\left(e_{3} \cdot n_{3}\right)\left(\mathcal{P}_{\mathcal{F}_{3}, N-1}^{N-1} \mid\left(\mathrm{F}_{N-3}^{0}(\bar{x}, w) \otimes e_{1} \otimes e_{2}\right)\right) \cdot v_{3}^{1} \\
& =\left(e_{3} \cdot n_{3}\right)\left(\mathrm{F}_{N-3}^{0}(\bar{x}, w) \otimes e_{1} \otimes e_{2}\right) \cdot v_{3}^{1} \\
& =\left(e_{3} \cdot n_{3}\right)\left(e_{2} \cdot v_{3}^{1}\right) \mathrm{F}_{N-3}^{0}(\bar{x}, w) \otimes e_{1} .
\end{aligned}
$$

Hence, as $\left(e_{1}\right)_{\perp \mathcal{L}_{1}}=0$, the term $\mathcal{K}_{f_{1}}\left(\mathcal{O}_{f_{1}}^{N} \mid\left(\mathrm{F}_{N-3}^{0}(\bar{x}, w) \otimes e_{1} \otimes e_{2} \otimes e_{3}\right)\right)$ vanishes. We continue our evaluations:

$\mathcal{O}_{f_{1}}^{N} \mid\left(\mathrm{F}_{N-2}^{1}\left(\bar{x}, f_{j}\right) \otimes E_{j}\right)=\sum_{k \neq 1}\left(\mathcal{P}_{\mathcal{F}_{k}}^{N-1} \mid\left(\left(\mathrm{F}_{N-2}^{1}\left(\bar{x}, f_{j}\right) \otimes e_{j+1} \otimes e_{j+2}\right) \cdot n_{k}\right)\right) \cdot v_{k}^{1}$

The terms in this sum vanish unless $k=j+2$. As also $k \neq 1$ the sum vanishes if $j=2$. Otherwise, using the fact that $e_{j}$ is tangential to $\mathcal{F}_{j+2}$, it reads

$$
\begin{aligned}
\mathcal{O}_{f_{1}}^{N} \mid & \left(\mathrm{F}_{N-2}^{1}\left(\bar{x}, f_{j}\right) \otimes E_{j}\right) \\
& =\left(e_{j+2} \cdot n_{j+2}\right)\left(\mathcal{P}_{\mathcal{F}_{j+2}}^{N-1} \mid\left(\mathrm{F}_{N-2}^{1}\left(\bar{x}, f_{j}\right) \otimes e_{j+1}\right)\right) \cdot v_{j+2}^{1} \\
& =\left(e_{j+2} \cdot n_{j+2}\right) \sum_{q=1}^{N-1}\left(\mathcal{P}_{\mathcal{F}_{j+2}, q}^{N-1} \mid\left(\mathrm{F}_{N-2}^{1}\left(\bar{x}, f_{j}\right) \otimes e_{j+1}\right)\right) \cdot v_{j+2}^{1} \\
& =\left(e_{j+2} \cdot n_{j+2}\right)\left(\mathcal{P}_{\mathcal{F}_{j+2}, N-1}^{N-1} \mid\left(\mathrm{F}_{N-2}^{1}\left(\bar{x}, f_{j}\right) \otimes e_{j+1}\right)\right) \cdot v_{j+2}^{1} \\
& =\left(e_{j+2} \cdot n_{j+2}\right)\left(e_{j+1} \cdot v_{j+2}^{1}\right) \mathrm{F}_{N-2}^{1}\left(\bar{x}, f_{j}\right) .
\end{aligned}
$$

If $j=3$ the term vanishes, as $e_{j+1} \cdot v_{j+2}^{1}=e_{1} \cdot v_{2}^{1}=0$. Thus the only non-vanishing term is for $j=1$ :

$$
\begin{aligned}
\mathcal{K}_{f_{1}}\left(\mathcal{O}_{f_{1}}^{N}\left(\mathrm{~F}_{N-2}^{1}\left(\bar{x}, f_{1}\right) \otimes E_{1}\right)\right) & =\left(e_{3} \cdot n_{3}\right)\left(e_{2} \cdot v_{3}^{1}\right) \mathcal{K}_{f_{1}}\left(\mathrm{~F}_{N-2}^{1}\left(\bar{x}, f_{1}\right)\right) \\
& =\left(e_{3} \cdot n_{3}\right)\left(e_{2} \cdot\left(n_{3} \times e_{1}\right)\right) \mathrm{F}_{N-2}^{1}\left(\bar{x}, f_{1}\right) \\
& =d \mathrm{~F}_{N-2}^{1}\left(\bar{x}, f_{1}\right) .
\end{aligned}
$$

In this way we have proven formula (13). 
The formula (13) is not, at this point, an actual representation result. Indeed, it is valid only for the particular shape $f_{1}$ and the way we introduced $\tilde{C}_{N}$ explicitly involves $\mathrm{F}_{N-2}^{1}\left(\bar{x}, f_{1}\right)$. However, if $f$ is the shape of the edge of some tetrahedron, we can with no loss of generality consider that $f=f_{1}$ with the notation of Section 2.2. From Lemma 4 we know that $\mathrm{F}_{N-2}^{1}(\bar{x}, f)=\mathcal{K}_{\mathcal{L}}\left(\mathcal{O}_{f}^{N} \mid \tilde{C}_{N}\right)$ and the technical Lemma 7, which for the sake of clarity we present in Appendix, establishes that this identity remains valid when we replace $\tilde{C}_{N}$ by its totally symmetric part $C_{N}$. As $C_{N}$ does not depend on any geometrical quantity, the following representation theorem holds:

Theorem 2. The tensor $C_{N}$ defined by Theorem 1 satisfies, for any edge $\mathcal{L}$ with shape $f$ of some non degenerated tetrahedron,

$$
\mathrm{F}_{N-2}^{1}(\bar{x}, f)=\mathcal{K}_{\mathcal{L}}\left(\mathcal{O}_{f}^{N} \mid C_{N}\right) .
$$

\subsection{Representation Theorem for Wedge Actions}

In order to describe the dependence of the wedge actions upon the shape of the wedge let us define the appropriated boundary operator.

Definition 4. We denote $[\hat{x}]$ the set of the subscripts of the three edges concurring on the wedge $\hat{x}$. Its shape $w$ is determined by the vectors $e_{j}(j \in[\hat{x}])$, which are the unit tangents to these edges pointing outward of $\hat{x}$. At $\hat{x}$, the boundary operator $\mathcal{O}_{w}$ is the tensor of order $2 p-3$ defined by setting, for any tensor $X$ of order $p$,

$$
\mathcal{O}_{w}^{p} \mid X:=\sum_{j \in[\hat{x}]}\left(\mathcal{P}_{\mathcal{L}_{j}}^{p-2} \mid\left(\mathcal{O}_{f_{j}}^{p} \mid X\right)\right) \cdot e_{j}
$$

where $\mathcal{P}_{\mathcal{L}_{j}}^{p-2}$ and $\mathcal{O}_{f_{j}}^{p}$ have been defined in the previous subsection.

Lemma 5. The tensor $\tilde{C}_{N}$ introduced by Lemma 2 also satisfies

$$
\mathrm{F}_{N-3}^{0}(\bar{x}, w)=\mathcal{K}\left(\mathcal{O}_{w}^{N} \mid \tilde{C}_{N}\right),
$$

$w$ denoting the shape of the wedge $\hat{x}$ of a tetrahedron $\Delta\left(\hat{x}, n_{1}, n_{2}, n_{3}, n_{0}, h_{0}\right)$.

Proof. We prove formula (15) by computing $\mathcal{O}_{w}^{N} \mid \tilde{C}_{N}$ in a way similar to the one we followed for edge actions. The term

$$
\begin{aligned}
\mathcal{O}_{w}^{N} \mid & \left(\mathrm{F}_{N-1}^{2}\left(\bar{x}, n_{i}\right) \otimes e_{i}\right) \\
& =\sum_{j=1}^{3}\left(\mathcal{P}_{\mathcal{L}_{j}}^{N-2} \mid\left(\sum_{k \neq j}\left(\mathcal{P}_{\mathcal{F}_{k}}^{N-1} \mid\left(\left(\mathrm{F}_{N-1}^{2}\left(\bar{x}, n_{i}\right) \otimes e_{i}\right) \cdot n_{k}\right) \cdot v_{k}^{j}\right)\right)\right) \cdot\left(-e_{j}\right) \\
& =-\left(e_{i} \cdot n_{i}\right) \sum_{j \neq i}\left(\mathcal{P}_{\mathcal{L}_{j}}^{N-2} \mid\left(\mathcal{P}_{\mathcal{F}_{i}}^{N-1} \mid\left(\mathrm{F}_{N-1}^{2}\left(\bar{x}, n_{i}\right)\right) \cdot v_{i}^{j}\right)\right) \cdot e_{j}
\end{aligned}
$$


vanishes, as $\mathrm{F}_{N-1}^{2}\left(\bar{x}, n_{i}\right)$ is completely orthogonal to $\mathcal{F}_{i}$. On the other hand, the term

$$
\begin{aligned}
& \mathcal{O}_{w}^{N} \mid\left(\mathrm{F}_{N-2}^{1}\left(\bar{x}, f_{i}\right) \otimes E_{i}\right) \\
& \left.=\sum_{j=1}^{3}\left(\mathcal{P}_{\mathcal{L}_{j}-2}^{N-2} \mid \sum_{k \neq j}\left(\mathcal{P}_{\mathcal{F}_{k}}^{N-1} \mid\left(\left(\mathrm{F}_{N-2}^{1}\left(\bar{x}, f_{i}\right) \otimes e_{i+1} \otimes e_{i+2}\right) \cdot n_{k}\right) \cdot v_{k}^{j}\right)\right)\right) \cdot\left(-e_{j}\right) \\
& =-\left(e_{i+2} \cdot n_{i+2}\right) \sum_{j \neq i+2}\left(\mathcal{P}_{\mathcal{L}_{j}}^{N-2} \mid\left(\mathcal{P}_{\mathcal{F}_{i+2}}^{N-1} \mid\left(\mathrm{F}_{N-2}^{1}\left(\bar{x}, f_{i}\right) \otimes e_{i+1}\right) \cdot v_{i+2}^{j}\right) \cdot e_{j}\right) \\
& =-\left(e_{i+2} \cdot n_{i+2}\right) \sum_{j \neq i+2}\left(e_{i+1} \cdot v_{i+2}^{j}\right)\left(\mathcal{P}_{\mathcal{L}_{j}}^{N-2} \mid\left(\mathrm{F}_{N-2}^{1}\left(\bar{x}, f_{i}\right)\right) \cdot e_{j}\right) \\
& =-\left(e_{i+2} \cdot n_{i+2}\right)\left(e_{i+1} \cdot v_{i+2}^{i}\right)\left(\mathcal{P}_{\mathcal{L}_{i}}^{N-2} \mid\left(\mathrm{F}_{N-2}^{1}\left(\bar{x}, f_{i}\right)\right) \cdot e_{i}\right)
\end{aligned}
$$

also vanishes, as $\mathrm{F}_{N-2}^{1}\left(\bar{x}, f_{i}\right)$ is completely orthogonal to $\mathcal{L}_{i}$. Finally we have $\mathcal{O}_{w}^{N} \mid\left(\mathrm{F}_{N-3}^{0}(\bar{x}, w) \otimes e_{1} \otimes e_{2} \otimes e_{3}\right)$

$$
\begin{aligned}
& =\sum_{j=1}^{3}\left(\mathcal{P}_{\mathcal{L}_{j}}^{N-2} \mid\left(\sum_{k \neq j}\left(\mathcal{P}_{\mathcal{F}_{k}}^{N-1} \mid\left(\left(\mathrm{F}_{N-3}^{0}(\bar{x}, w) \otimes e_{1} \otimes e_{2} \otimes e_{3}\right) \cdot n_{k}\right) \cdot v_{k}^{j}\right)\right)\right) \cdot\left(-e_{j}\right) \\
& =-\left(e_{3} \cdot n_{3}\right) \sum_{j=1}^{2}\left(\mathcal{P}_{\mathcal{L}_{j}}^{N-2} \mid\left(\left(\mathcal{P}_{\mathcal{F}_{3}}^{N-1} \mid\left(\left(\mathrm{F}_{N-3}^{0}(\bar{x}, w) \otimes e_{1} \otimes e_{2}\right)\right) \cdot v_{3}^{j}\right)\right)\right) \cdot e_{j} \\
& =-\left(e_{3} \cdot n_{3}\right) \sum_{j=1}^{2}\left(\mathcal{P}_{\mathcal{L}_{j}}^{N-2} \mid\left(\left(\mathrm{F}_{N-3}^{0}(\bar{x}, w) \otimes e_{1} \otimes e_{2}\right) \cdot v_{3}^{j}\right)\right) \cdot e_{j} \\
& =-\left(e_{3} \cdot n_{3}\right)\left(e_{2} \cdot v_{3}^{1}\right)\left(\left(\mathcal{P}_{\mathcal{L}_{1}}^{N-2} \mid\left(\left(\mathrm{F}_{N-3}^{0}(\bar{x}, w) \otimes e_{1}\right)\right)\right) \cdot e_{1}\right) \\
& =-\left(e_{3} \cdot n_{3}\right)\left(e_{2} \cdot v_{3}^{1}\right) \mathrm{F}_{N-3}^{0}(\bar{x}, w) \\
& =-\left(e_{3} \cdot n_{3}\right)\left(e_{2} \cdot\left(n_{3} \times e_{1}\right)\right) \mathrm{F}_{N-3}^{0}(\bar{x}, w) \\
& =-d \mathrm{~F}_{N-3}^{0}(\bar{x}, w),
\end{aligned}
$$

which concludes the proof.

Again, formula (15) cannot be considered as a representation result. Indeed, it is valid only for the particular shape $w$ and the definition of $\tilde{C}_{N}$ explicitly involves $\mathrm{F}_{N-3}^{0}(\bar{x}, w)$. However, any wedge shape $w$ can be considered as the wedge at the vertex $\bar{x}$ of a tetrahedron with the notation defined in Section 2.2. Applying Lemma 5, we know that formula (15) holds. The technical Lemma 8 which we present in Appendix allows us to replace $\tilde{C}_{N}$ by $C_{N}$. We thus get the representation result:

Theorem 3. The tensor $C_{N}$ defined by Theorem 1 satisfies, for any wedge shape $w$ of some non degenerated tetrahedron,

$$
\mathrm{F}_{N-3}^{0}(\bar{x}, w)=\mathcal{K}\left(\mathcal{O}_{w}^{N} \mid C_{N}\right)
$$




\section{Extension to More General Shapes}

\subsection{Domains and Shapes}

At this point we have obtained representation theorems valid only for tetrahedrons. We have to extend them to a larger class of domains if we want to use the theory in the practical cases considered by mechanicians. We do not intend to search for the largest class which could be treated. Indeed, this is already a very difficult problem for the classical Cauchy theory. For instance we do not treat non-convex polyhedral domains or vertex where more than three edges concur. However the class of domains to which our results can apply may likely be considerably enlarged by playing with unions and intersections of domains. More precisely:

Definition 5. the domains which we consider as admissible are those domains $D$ such that, for any $x \in D$, there exist a tetrahedron $\Delta$, a $C^{1}$ diffeomorphism $\Phi$ and a neighborhood $O_{x}$ of $x$ such that

$$
\Phi(\Delta) \cap O_{x}=D \cap O_{x} .
$$

As we are interested in the dependence of contact interactions on the shape of the domain, we need to define precisely what we mean by shape. We first say that the shape of a domain $D$ at the point $x$ is the same as the shape of the domain $D^{\prime}$ at the point $x^{\prime}$ if and only if there exists a neighborhood $O_{x}$ of $x$ and a translation $t_{x-x^{\prime}}$ such that

$$
t_{x-x^{\prime}}\left(x^{\prime}\right)=x, \text { and } t_{x-x^{\prime}}\left(D^{\prime}\right) \cap O_{x}=D \cap O_{x} .
$$

This makes an equivalence relation. Thus we can set:

Definition 6. We call shape of $D$ at the point $x$ the equivalence class with respect to the above defined equivalence relation. We denote this equivalence class by means of the symbol $\widetilde{(D, x)}$.

This notion of shape is local and, according to the definition, when a surface is rotated its shape changes. We assume that the densities $\mathrm{F}_{k}^{q}$ in $\mathfrak{S}(B, U)$ depend on the sub-body $B$ only through its shape at point $x: \mathrm{F}_{k}^{q}=\mathrm{F}_{k}^{q}(x, \widetilde{(B, x)})$. Note that this assumption is coherent with the assumptions made in the previous section where the considered sub-bodies were limited to tetrahedrons.

Definition 7. Let $D$ and $D^{\prime}$ two admissible domains. We say that the two shapes $\widetilde{(D, x)}$ and $\left.\widetilde{\left(D^{\prime}, x^{\prime}\right.}\right)$ are tangent if there exists a $C^{1}$ diffeomorphism $\Phi$ satisfying $\phi(x)=x^{\prime}, D \Phi(x)=I d$ and a neighborhood $O_{x^{\prime}}$ of $x^{\prime}$ such that

$$
\Phi(D) \cap O_{x^{\prime}}=D^{\prime} \cap O_{x^{\prime}} .
$$

This is clearly an equivalence relation and the shapes of the admissible domains are, by definition, tangent to some shape of a tetrahedron. This enables us to divide the boundary $\partial D$ of an admissible domain in the sets $\partial_{2} D$ of points $x$ where $\widetilde{(D, x)}$ is tangent to a face shape, $\partial_{1} D$ where $\widetilde{(D, x)}$ is tangent to a edge shape and $\partial_{0} D$ where $\widetilde{(D, x)}$ is tangent to a wedge shape of some tetrahedron. 
Unlike NoLL [34], we do not assume the uniform boundedness of the densities with respect to all possible shapes. Such a strong assumption would kill all possibilities of describing stress states of order larger than one.

In order to weaken this assumption we need to define what we call a set of prescribed set of shapes. We first remark that the shape at $x$ of the domain $D \cap$ $\{y,(y-x) \cdot u<0\}$ depends only on the shape at $x$ of the domain $D$ and on $u$. We denote it $\operatorname{cut}(\widehat{(D, x)}, u)$.

Definition 8. We say that $E$ is prescribed set of shapes if there exists a finite sequence $\left(D_{i}\right)$ of admissible domains and a finite sequence $\left(u_{j}\right)$ of unit vectors such that any shape $f$ in $E$ satisfies $f=\widetilde{\left(D_{i}, x\right)}$ or $f=\operatorname{cut}\left(\widetilde{\left(D_{i}, x\right)}, u_{j}\right)$ or $\left.f=\operatorname{cut}\left(\operatorname{cut}\left(\widetilde{\left(D_{i}, x\right.}\right), u_{j}\right), u_{k}\right)$ for some point $x$ and some $i, j, k .{ }^{6}$

For any domain $D$ and any unit vectors $u, v$, we assume the continuity $: \forall \xi>0$, $\forall \bar{x} \in \partial_{i} D, \exists \eta>0$ such that $\forall x \in \partial_{i} D$ satisfying $\|x-\bar{x}\|<\eta$

$$
\begin{aligned}
& \left\|\mathrm{F}_{q}^{i}(x, \widetilde{(D, x)})-\mathrm{F}_{q}^{i}(\bar{x}, \widetilde{(D, \bar{x})})\right\|<\xi, \\
& \| \mathrm{F}_{q}^{i}\left(x, \operatorname{Cut}(\widetilde{(D, x)}, u)-\mathrm{F}_{q}^{i}(\bar{x}, \operatorname{Cut}(\widetilde{(D, \bar{x})}, u) \|<\xi,\right. \\
& \| \mathrm{F}_{q}^{i}\left(x, \operatorname{Cut}(\operatorname{Cut}(\widetilde{(D, x)}, u), v)-\mathrm{F}_{q}^{i}(\bar{x}, \operatorname{Cut}(\operatorname{Cut}(\widetilde{(D, \bar{x})}, u), v) \|<\xi,\right.
\end{aligned}
$$

and the equicontinuity on a prescribed set of shapes $E: \forall \xi>0, \forall \bar{x}, \exists \eta>0$ such that $\forall x, \forall f \in E$,

$$
\|x-\bar{x}\|<\eta \Rightarrow\left\|\mathrm{F}_{q}^{i}(x, f)-\mathrm{F}_{q}^{i}(\bar{x}, f)\right\|<\xi
$$

where $i=2,1$ or 0 depending on if $f$ is a regular shape, an edge or a wedge shape.

\subsection{Generalization of Noll's Theorem}

We prove here a theorem analogous to NoLL's theorem [34] which states that the highest order terms of the stress state depend on the shape of the domain only through the tangent shape. Assumption (19), and therefore our definition of prescribed shapes, play an essential role here.

Theorem 4. If an admissible domain $D$ is tangential at some point $\bar{x} \in \partial_{q} D$ for some $q \in\{0,1,2\}$ to a tetrahedron $\Delta$, then

$$
\mathrm{F}_{N+1-q}^{q}(\bar{x}, \widetilde{(D, \bar{x})})=\mathrm{F}_{N+1-q}^{q}(\bar{x}, \widetilde{(\Delta, \bar{x})}) .
$$

${ }^{6}$ For example, the shapes of the family of cubes $C_{t}:=[0, t]^{3}$, for $t \in(0,1]$, constitute a prescribed set of shapes as any of these shapes is identical to some shape of the unit cube $C_{1}$. On the contrary, the shapes of the family of cubes $C_{t}^{\prime}$ which are the images of $[0,1]^{3}$ under a rotation of angle $t$, for $t \in(0,1]$, around an axis $u$, do not constitute a prescribed set of shapes. Analogously, the shapes of the family of spheres $S_{t}$ of center 0 and radius $t$ do not constitute a prescribed set of shapes. 
Proof. We start by considering the case when $\bar{x}$ is a regular point of a face of $D$ $(q=2)$. We denote $\bar{n}$ as the outward unit normal to the boundary at $\bar{x}$. As $\Delta$ is tangential to $D$ at $\bar{x}$, the shape of $\Delta$ at $\bar{x}$ is the plane shape $\bar{n}$. Let us temporarily use an orthonormal coordinate system $\left(\bar{x}, b_{1}, b_{2}, \bar{n}\right)$ and consider the family of parallelepipeds:

$$
\square_{\varepsilon}=[0, \varepsilon] \times[0, \varepsilon] \times\left[-\xi_{\varepsilon}, \xi_{\varepsilon}\right] .
$$

Let us define the sets:

$$
D_{\varepsilon}:=D \cap \square_{\varepsilon}, \quad \mathcal{F}_{\varepsilon}:=\partial D \cap \square_{\varepsilon} \text { and } \quad \mathcal{F}_{-}:=\left\{x \in \square_{\varepsilon} ; x_{3}=-\xi_{\varepsilon}\right\} .
$$

As $\Delta$ is tangential to $D$ at $\bar{x}$, a $\xi_{\varepsilon}$ of order $o(\varepsilon)$ can be found such that, for $\varepsilon$ sufficiently small, $\mathcal{F}_{\varepsilon}$ does not intersect the face $\mathcal{F}_{-}$. Clearly, the shapes of the boundary of $D_{\varepsilon}$ for all $\varepsilon$ make a set of prescribed shapes.

We apply to the family of domains $D_{\varepsilon}$ the quasi-balance inequality with the fixed test field $U(x)=(x \cdot \bar{n})^{N-1} U_{0}$. It implies that the limit

$$
\lim _{\varepsilon \longrightarrow 0} \varepsilon^{-2}\left(\int_{\mathcal{F}_{\varepsilon}} F_{N-1}^{2}(x, \widetilde{(D, x)})\left|\bar{n}^{\otimes N-1} U_{0}+\int_{\mathcal{F}_{-}} F_{N-1}^{2}(x,-\bar{n})\right| \bar{n}^{\otimes N-1} U_{0}\right)
$$

vanishes. Indeed: (1) all other surface terms in the expression of the stress state are negligible as the areas of the lateral faces of $D_{\varepsilon}$ are of order $o\left(\varepsilon^{2}\right)$, (2) the edge terms are negligible as the lengths of the edges are of order $\varepsilon$ and the fields $\nabla^{q} U$ are of order $o\left(\varepsilon^{N-1-q}\right)$, (3) this order of magnitude of the fields $\nabla^{q} U$ also makes the wedge terms negligible. This leads to

$$
\begin{aligned}
\left.\mathrm{F}_{N-1}^{2}(\bar{x}, \widetilde{(D, \bar{x}})\right) \mid \bar{n}^{\otimes N-1} & =-\mathrm{F}_{N-1}^{2}(\bar{x},-\bar{n}) \mid \bar{n}^{\otimes N-1} \\
& =-\left(C_{N}(\bar{x}) \mid(-\bar{n})^{\otimes N}\right)(-1)^{N-1},
\end{aligned}
$$

and so

$$
\mathrm{F}_{N-1}^{2}(\bar{x}, \widetilde{(D, \bar{x})})=\left(C_{N}(\bar{x}) \mid \bar{n}^{\otimes N}\right) \otimes \bar{n}^{\otimes N-1}=\mathrm{F}_{N-1}^{2}(\bar{x}, \bar{n}) .
$$

Now we consider the case when $\bar{x} \in \partial_{1}(D)$. Consider $\Delta$ tangential to $D$ at $\bar{x}$ and $\Phi$ the diffeomorphism given by Definition 7. The point $\bar{x}$ is an internal point of an edge $\mathcal{L}_{1}$ of $\Delta$ where two faces $\mathcal{F}_{2}, \mathcal{F}_{3}$ join. We denote $\bar{e}_{1}$ as a unit vector tangential to the edge and $\bar{n}_{2}, \bar{n}_{3}$ the normals to the faces. We introduce a vector $e$ orthogonal to $\bar{e}_{1}$ and satisfying

$$
e \cdot \bar{n}_{2}>0, \quad e \cdot \bar{n}_{3}>0 .
$$

We temporarily use the orthonormal coordinate system $\left(\bar{e}_{1}, b, e\right)$ and use in this system of coordinates the same notation for $\square_{\varepsilon}$. We introduce $t_{\varepsilon}$ as the translation of vector $\xi_{\varepsilon} e$, and we redefine

$$
D_{\varepsilon}:=t_{\varepsilon}\left(D \cap \square_{\varepsilon}\right), \quad \mathcal{F}_{\varepsilon}:=t_{\varepsilon}\left(\partial D \cap \square_{\varepsilon}\right)
$$

and

$$
\mathcal{F}_{-}:=t_{\varepsilon}\left(\left\{x \in \square_{\varepsilon} ; x_{3}=-\xi_{\varepsilon}\right\}\right)
$$


We moreover introduce the sets

$\mathcal{L}_{\varepsilon}:=t_{\varepsilon}\left(\Phi\left(\mathcal{L}_{1}\right) \cap \square_{\varepsilon}\right), \quad \mathcal{F}_{2, \varepsilon}:=t_{\varepsilon}\left(\Phi\left(\mathcal{F}_{2}\right) \cap \square_{\varepsilon}\right), \quad \mathcal{F}_{3, \varepsilon}:=t_{\varepsilon}\left(\Phi\left(\mathcal{F}_{3}\right) \cap \square_{\varepsilon}\right)$.

As $\Delta$ is tangential to $D$ at $\bar{x}$, a $\xi_{\varepsilon}=o(\varepsilon)$ can be found such that, for $\varepsilon$ sufficiently small, the line $\mathcal{L}_{\varepsilon}$ does not intersect the face $\mathcal{F}_{-}$. The shapes of $D_{\varepsilon}$ for all $\varepsilon$ still belong to a set of prescribed shapes. Quasi-balance Inequality, when applied with the fixed test field $U(x)=((x-\bar{x}) \cdot e)^{N-1} U_{0}$ to the family of domains $D_{\varepsilon}$, implies

$$
\begin{gathered}
\lim _{\varepsilon \longrightarrow 0}\left(\xi_{\varepsilon} \varepsilon\right)^{-1}\left(\int_{\mathcal{F}_{\varepsilon}} F_{N-1}^{2}\left(\bar{x}, \widehat{\left(D_{\varepsilon}, \bar{x}\right.}\right)\right)\left|e^{\otimes N-1} U_{0}+\int_{\mathcal{F}_{-}} \mathrm{F}_{N-1}^{2}(\bar{x},-e)\right| e^{\otimes N-1} U_{0} \\
\left.+\int_{\mathcal{L}_{\varepsilon}} \mathrm{F}_{N-2}^{1}\left(\bar{x}, \widehat{\left(D_{\varepsilon}, x\right)}\right) \mid e^{\otimes N-2}((x-\bar{x}) \cdot e) U_{0}\right)=0 .
\end{gathered}
$$

Indeed: (1) all the other surface terms in the stress state expression are negligible as the areas of the faces $\left\{x_{1}= \pm \varepsilon\right\}$ are of order $\xi_{\varepsilon}^{2} ;(2)$ the other edge terms are negligible as either their lengths are of the order $\xi_{\varepsilon}$ and the field $\nabla^{k} U$ with $k<N-1$ is of order smaller or equal to $\xi_{\varepsilon}$ or they lay in the plane $(x-\bar{x}) \cdot e=0$; (3) the wedge terms are also negligible as $\nabla^{k} U$ with $k<N-2$ is of order smaller or equal to $\xi_{\varepsilon}^{2}$. We get

$$
\begin{aligned}
\lim _{\varepsilon \longrightarrow 0}\left(\frac{\left|\mathcal{F}_{2, \varepsilon}\right|}{\xi_{\varepsilon} \varepsilon}\right) & \mathrm{F}_{N-1}^{2}\left(\bar{x}, \bar{n}_{2}\right)\left|e^{\otimes N-1}+\lim _{\varepsilon \longrightarrow 0}\left(\frac{\left|\mathcal{F}_{3, \varepsilon}\right|}{\xi_{\varepsilon} \varepsilon}\right) \mathrm{F}_{N-1}^{2}\left(\bar{x}, \bar{n}_{3}\right)\right| e^{\otimes N-1} \\
& +\lim _{\varepsilon \longrightarrow 0}\left(\frac{\left|\mathcal{F}_{-}\right|}{\xi_{\varepsilon} \varepsilon}\right) \mathrm{F}_{N-1}^{2}(\bar{x},-e) \mid e^{\otimes N-1} \\
& +\lim _{\varepsilon \rightarrow 0}\left(\frac{\left|\mathcal{L}_{\varepsilon}\right| \xi_{\varepsilon}}{\xi_{\varepsilon} \varepsilon}\right) \mathrm{F}_{N-2}^{1}(\bar{x}, \widehat{(D, \bar{x})}) \mid e^{\otimes N-2}=0 .
\end{aligned}
$$

Computing the limits in the previous equality is straightforward. We have

$$
\begin{aligned}
& \frac{1}{v_{2}^{1} \cdot e} \mathrm{~F}_{N-1}^{2}\left(\bar{x}, \bar{n}_{2}\right)\left|e^{\otimes N-1}+\frac{1}{v_{3}^{1} \cdot e} \mathrm{~F}_{N-1}^{2}\left(\bar{x}, \bar{n}_{3}\right)\right| e^{\otimes N-1} \\
& \quad+\left(\frac{n_{2} \cdot e}{v_{2}^{1} \cdot e}+\frac{n_{3} \cdot e}{v_{3}^{1} \cdot e}\right) \mathrm{F}_{N-1}^{2}(\bar{x},-e)\left|e^{\otimes N-1}+\mathrm{F}_{N-2}^{1}(\bar{x}, \widetilde{(D, \bar{x})})\right| e^{\otimes N-2}=0 .
\end{aligned}
$$

Therefore, the quantity $\mathrm{F}_{N-2}^{1}(\bar{x}, \widetilde{(D, \bar{x})}) \mid e^{\otimes N-2}$ depends on the shape of $D$ only through vectors $e, \bar{n}_{2}$ and $\bar{n}_{3}$. The vector $e$ is generic in the plane cone defined by (22), we invoke Lemma 2 in the case $d=2$ to conclude that the plane tensor $\mathrm{F}_{N-2}^{1}(\bar{x}, \widehat{(D, \bar{x})})$ depends only on $\bar{n}_{2}$ and $\bar{n}_{3}$, that is, on the geometry of the tangent tetrahedron.

The case when $\bar{x}$ is a wedge point of $D(q=0)$ is treated in a very similar way. Now three faces of $D$ and three edges are joining at $\bar{x}$. The tetrahedron $\Delta$ is tangent to $D$ at a wedge point $\bar{x}$ where three faces of normals $\bar{n}_{1}, \bar{n}_{2}, \bar{n}_{3}$ join. We introduce a vector $e$ satisfying

$$
e \cdot \bar{n}_{1}>0, \quad e \cdot \bar{n}_{2}>0, \quad e \cdot \bar{n}_{3}>0
$$


and use notations compatible with the previous ones in an orthonormal coordinate system $\left(\bar{x}, b_{1}, b_{2}, e\right)$. Applying the quasi balance of power for the same test field and with the family of domains defined in (23). We get

$$
\begin{aligned}
\sum_{j=1}^{3} \lim _{\varepsilon \longrightarrow 0} & \left(\frac{\left|\mathcal{F}_{j, \varepsilon}\right|}{\xi_{\varepsilon}^{2}}\right)\left(\mathrm{F}_{N-1}^{2}\left(\bar{x}, \bar{n}_{j}\right) \mid e^{\otimes N-1}\right) \\
& +\lim _{\varepsilon \longrightarrow 0}\left(\frac{\left|\mathcal{F}_{-}\right|}{\xi_{\varepsilon}^{2}}\right)\left(\mathrm{F}_{N-1}^{2}(\bar{x},-e) \mid e^{\otimes N-1}\right) \\
& \left.+\sum_{k=1}^{3} \lim _{\varepsilon \longrightarrow 0}\left(\left|\mathcal{L}_{k, \varepsilon}\right|\right) \mathrm{F}_{N-2}^{1}(\bar{x}, \widetilde{(D, \bar{x}})\right) \mid e^{\otimes N-2} \\
& \left.+\left(\mathrm{F}_{N-3}^{0}(\bar{x}, \widetilde{(D, \bar{x}})\right) \mid e^{\otimes N-3}\right)=0 .
\end{aligned}
$$

Computing the previous limits is again straightforward. They depend only on the vectors $e, \bar{n}_{1}, \bar{n}_{2}$ and $\bar{n}_{3}$. Therefore the quantity $\mathrm{F}_{N-3}^{0}(\bar{x}, \widetilde{(D, \bar{x})}) \mid e^{\otimes N-3}$ depends only on these vectors. As the vector $e$ is generic in the cone defined by (24), we invoke Lemma 2 in the case $d=3$ to conclude that the plane tensor $\mathrm{F}_{N-3}^{0}(\bar{x}, \widetilde{(D, \bar{x})})$ depends only on $\bar{n}_{1} \bar{n}_{2}$ and $\bar{n}_{3}$, that is, on the geometry of the tangent tetrahedron.

\section{An Algorithm for the Representation of Lower Order Contact Interactions}

In order to represent lower order contact interactions in a body submitted to a stress state of order $N$, we need the domains and action fields to satisfy stronger regularity assumptions.

From now on, we restrict our attention to domains which are locally diffeomorphic to a tetrahedron through a diffeomorphism of class $C^{N}$ and such that, for $q \in\{0,1,2\}, \partial_{q} D$ is a finite union of $q$-dimensional $C^{N}$ compact manifolds. Therefore, $I_{0}(D), I_{1}(D)$ and $I_{2}(D)$, denoting respectively the sets of labels of the elements of dimension $0,1,2$ (wedges, edges, faces), which are parts of the boundary of $D$, we write

$$
\partial_{0} D:=\cup_{\ell \in I_{0}(D)}\left\{x_{\ell}\right\}, \quad \partial_{1} D:=\cup_{\ell \in I_{1}(D)}\left\{\mathcal{L}_{\ell}\right\}, \quad \partial_{2} D:=\cup_{\ell \in I_{2}(D)}\left\{\mathcal{F}_{\ell}\right\} .
$$

We also assume that the stress state of order $N$ is smooth. More precisely the fields $\mathrm{F}_{k}^{i}$ are $C^{k}$ fields on the manifolds where they are defined.

These assumptions are needed as we will repeatedly use on each element of the boundary of $D$ the following divergence theorem: for any $C^{2}$ manifold with boundary $M$, and any $C^{1}$ tensor fields $X$ and $Y$ ( $X$ having an order greater than $Y$ ) defined on $M$ we have

$$
\int_{M}\left(X \cdot \Pi_{M}\right)\left|\nabla Y=-\int_{M}\left(\operatorname{div}_{M}\left(X \cdot \Pi_{M}\right)\right)\right| Y+\int_{\partial M}(X \cdot v) \mid Y
$$


where $\operatorname{div}_{M}(X)$ stands for the standard divergence operator on the manifold $M$ and $v$ denotes the unit vector orthogonal to $\partial M$, tangent to $M$ and external to it. ${ }^{7}$

The algorithm for the representation of lower order contact interactions is based on the following remark:

Lemma 6. Let $C_{N}$ denote the completely symmetric tensor which represents the highest order contact interactions $\mathrm{F}_{N-1}^{2}, \mathrm{~F}_{N-2}^{1}$ and $\mathrm{F}_{N-3}^{0}$ of some smooth stress state of order $N$. Then the quantity

$$
\begin{gathered}
\int_{D}\left(C_{N}\left|\nabla^{N} U+\operatorname{div}\left(C_{N}\right)\right| \nabla^{N-1} U\right)-\int_{\partial_{2} D} \mathrm{~F}_{N-1}^{2} \mid \nabla^{N-1} U \\
-\int_{\partial_{1} D} \mathrm{~F}_{N-2}^{1}\left|\nabla^{N-2} U-\int_{\partial_{0} D} \mathrm{~F}_{N-3}^{0}\right| \nabla^{N-3} U
\end{gathered}
$$

is a smooth stress state of order $N-1$.

Proof. The way we have constructed $C_{N}$, shows that it is of class $C^{N-1}$. The divergence theorem states that

$$
\int_{D}\left(C_{N}\left|\nabla^{N} U+\operatorname{div}\left(C_{N}\right)\right| \nabla^{N-1} U\right)=\int_{\partial_{2} D}\left(C_{N} \cdot n\right) \mid \nabla^{N-1} U .
$$

The right hand side of this identity can be decomposed using formula (33) and the representation formula (10). It reads

$$
\int_{\partial_{2} D} \mathrm{~F}_{N-1}^{2}\left|\nabla^{N-1} U+\int_{\partial_{2} D}\left(\mathcal{P}_{\partial_{2} D}^{N-1} \mid\left(C_{N} \cdot n\right)\right)\right| \nabla^{N-1} U .
$$

Owing to (32) we can compute the last addend of this sum by using the surface divergence theorem on each face of $D$. Defining

$$
\mathrm{G}_{N-2}^{2}:=\operatorname{div}_{\partial_{2} D}\left(\mathcal{P}_{\partial_{2} D}^{N-1} \mid\left(C_{N} \cdot n\right)\right)
$$

(which is of class $C^{N-2}$ ), it becomes

$$
-\int_{\partial_{2} D} \mathrm{G}_{N-2}^{2}\left|\nabla^{N-2} U+\sum_{j \in I_{1}(D)} \int_{\mathcal{L}_{j}}\left(\mathcal{O}_{f_{j}}^{N} \mid C_{N}\right)\right| \nabla^{N-2} U .
$$

Using formula (33) and the representation formula (14), the last addend in the previous sum can be decomposed in

$$
\sum_{j \in I_{1}(D)} \int_{\mathcal{L}_{j}} \mathrm{~F}_{N-2}^{1}\left|\nabla^{N-2} U+\sum_{j \in I_{1}(D)} \int_{\mathcal{L}_{j}}\left(\mathcal{P}_{\mathcal{L}_{j}}^{N-2} \mid\left(\mathcal{O}_{f_{j}}^{N} \mid C_{N}\right)\right)\right| \nabla^{N-2} U .
$$

7 A quick way for defining $\operatorname{div}_{M}(X)$ is to use any smooth extension of $X$ in the vicinity of the manifold and set $\operatorname{div}_{M}(X):=\nabla X \mid \Pi_{M}$ and to remark thereafter that the result of this operation depends only on the values of $X$ on the manifold. 
Owing again to (32), we finally apply the divergence theorem along each line $\mathcal{L}_{j}$. Setting on each line $\mathcal{L}_{j}, \mathrm{G}_{N-3}^{1}:=\operatorname{div}_{\mathcal{L}_{j}}\left(\mathcal{P}_{\mathcal{L}_{j}}^{N-2} \mid\left(\mathcal{O}_{f_{j}}^{N} \mid C_{N}\right)\right)$, we write the last quantity as the sum

$$
-\sum_{j \in I_{1}(D)} \int_{\mathcal{L}_{j}} \mathrm{G}_{N-3}^{1}\left|\nabla^{N-3} U+\sum_{\hat{x} \in I_{0}(D)} \mathrm{F}_{N-3}^{0}\right| \nabla^{N-3} U
$$

Collecting all these results, we obtain that the quantity defined in Lemma 6 can be written in the form

$$
-\int_{\partial_{2} D} \mathrm{G}_{N-2}^{2}\left|\nabla^{N-2} U-\int_{\partial_{1} D} \mathrm{G}_{N-3}^{1}\right| \nabla^{N-3} U
$$

which corresponds to a new smooth stress state of order $N-1$.

As $\mathrm{G}_{N-2}^{2}$ and $\mathrm{G}_{N-3}^{1}$ are not necessarily orthogonal to the shape where they are applied, the stress state of order $N-1$ given by this lemma has to be rewritten in the canonical form

$$
\sum_{k=0}^{N-2} \int_{\partial_{2} D} \tilde{\mathrm{F}}_{k}^{2}\left|\nabla_{\perp}^{k} U+\sum_{k=0}^{N-3} \int_{\partial_{1} D} \tilde{\mathrm{F}}_{k}^{1}\right| \nabla_{\perp}^{k} U+\sum_{k=0}^{N-4} \int_{\partial_{0} D} \tilde{\mathrm{F}}_{k}^{0} \mid \nabla^{k} U,
$$

where the $k$-forces $\tilde{F}_{k}^{i}$ can be made explicit in terms of $\mathrm{G}_{N-2}^{2}$ and $\mathrm{G}_{N-3}^{1}$.

The quantity $\int_{D}\left(C_{N}\left|\nabla^{N} U+\operatorname{div}\left(C_{N}\right)\right| \nabla^{N-1} U\right)$, being a volume integral, is by itself clearly quasi-balanced. Therefore, by summation, the new stress state defined by

$$
\begin{aligned}
\mathfrak{S}^{\prime}(D, U): & =\sum_{k=0}^{N-2} \int_{\partial_{2} D}\left(\mathrm{~F}_{k}^{2}+\tilde{\mathrm{F}}_{k}^{2}\right)\left|\nabla^{k} U+\sum_{k=0}^{N-3} \int_{\partial_{1} D}\left(\mathrm{~F}_{k}^{1}+\tilde{\mathrm{F}}_{k}^{1}\right)\right| \nabla^{k} U \\
& +\sum_{k=0}^{N-4} \int_{\partial_{0} D}\left(\mathrm{~F}_{k}^{0}+\tilde{\mathrm{F}}_{k}^{0}\right) \mid \nabla^{k} U,
\end{aligned}
$$

which is of order $N-1$ is also smooth and quasi-balanced. The representation theorems can be applied to the highest order terms of this new stress state: there exists a completely symmetric tensor $C_{N-1}$ of order $N-1$ such that

$$
\begin{gathered}
\mathrm{F}_{N-2}^{2}+\tilde{\mathrm{F}}_{N-2}^{2}=\mathcal{O}_{n}^{N-1} \mid C_{N-1}, \quad \mathrm{~F}_{N-3}^{1}+\tilde{\mathrm{F}}_{N-3}^{1}=\mathcal{K}_{\mathcal{L}}\left(\mathcal{O}_{f}^{N-1} \mid C_{N-1}\right) \\
\mathrm{F}_{N-4}^{0}+\tilde{\mathrm{F}}_{N-4}^{0}=\mathcal{K}\left(\mathcal{O}_{w}^{N-1} \mid C_{N-1}\right) .
\end{gathered}
$$

As the interactions $G$ and therefore $\tilde{F}$ can be made explicit, we have obtained a representation for $\mathrm{F}_{N-2}^{2}, \mathrm{~F}_{N-3}^{1}$ and $\mathrm{F}_{N-4}^{0}$. Clearly, this operation can be repeated in order to get a representation of all terms in the stress state through a chain of $N$ completely symmetric tensors with decreasing orders and regularity. 
We warn the reader that the chain of tensors $\left(C_{1}, \ldots, C_{N}\right)$ obtained in this way is equivalent but not identical to the one used in $[13,31,38]$ and denoted $(\tau, \ldots, \stackrel{N}{\tau})$ by Mindlin. This last one is more natural when one starts from the principle of virtual work. We have the relation $\stackrel{N}{\tau}=C_{N}$ and, for $1 \leqq q<N, \frac{q}{\tau}=C_{q}-\operatorname{div}\left(C_{q+1}\right)$. It happens that, even when following Mindlin, the boundary conditions are written in a more compact form when using $\left(C_{1}, \ldots, C_{N}\right)$.

We do not try to be explicit about the representation of all terms. Indeed, such a task needs the introduction of a very heavy notation. We restrict ourselves in Section 6 to the illustration of this algorithm by describing the representation of stress states of order one, two or three.

\section{First, Second and Third Gradient Theories}

The fact that our results enable us to recover the classical Cauchy theory is an evidence as we have closely followed the path of Cauchy. After quickly checking it, we verify here that they also enable us to recover the now widely used second gradient theory or the third gradient theory described in [31].

\subsection{Cauchy First Gradient Theory}

When $N=1$, the stress state is reduced to

$$
\mathfrak{S}(D, U)=\int_{\partial_{2} D} \mathrm{~F}_{0}^{2} \mid U .
$$

This corresponds to the Cauchy postulate that contact interactions can be described by a surface density of forces distributed along the regular part of the boundary. Our theorem reduces to the theorem established by Cauchy. Indeed, as $\mathcal{O}_{n}^{1}=n$, our theorem states that there exists a tensor $C_{1}$ (the Cauchy stress tensor) of order 1 such that $\mathrm{F}_{0}^{2}(x, n)=C_{1}(x) \cdot n$. The reader should not feel outraged by the fact that the Cauchy stress tensor is of order one. We recall that we have made no assumption on the tensorial nature of $U$ and considered it in our calculation as a scalar. So are the dual quantities $\mathrm{F}_{0}^{2}$. If $U$ is a vector in the physical space, the theorem can be applied component-wise and the Cauchy stress tensor becomes a tensor of order two. Note that the complete symmetry stated in our theorem is irrelevant in the case $N=1$ and that such a symmetry is of quite different nature from the well known symmetry of the Cauchy stress tensor. This latter is a consequence of assumptions (physical nature of the kinematic descriptor and Galilean invariance) which are out of the scope of our considerations.

\subsection{Second Gradient Theory}

When $N=2$, the stress state reads

$$
\mathfrak{S}(D, U)=\int_{\partial_{2} D} \mathrm{~F}_{0}^{2}\left|U+\mathrm{F}_{1}^{2}\right| \nabla_{\perp} U+\int_{\partial_{1} D} \mathrm{~F}_{0}^{1} \mid U .
$$


Let us make explicit the boundary operators when $N=2$. We have $\mathcal{O}_{n}^{2}=n^{\otimes 3}$ and $\mathcal{O}_{f_{j}}^{2}=\sum_{k \in\left[\mathcal{L}_{j}\right]} v_{k}^{j} \otimes n_{k}$. Indeed,

$$
\left(\mathcal{O}_{f_{j}}^{2}\right)_{i_{1}, i_{2}}:=\sum_{k \in\left[\mathcal{L}_{j}\right]}\left(v_{k}^{j}\right)_{\ell}\left(\mathcal{P}_{\mathcal{F}_{k}}^{1}\right)_{\ell, i_{1}}\left(n_{k}\right)_{i_{2}}=\sum_{k \in\left[\mathcal{L}_{j}\right]}\left(v_{k}^{j}\right)_{\ell}\left(\Pi_{\mathcal{F}_{k}}\right)_{\ell, i_{1}}\left(n_{k}\right)_{i_{2}} .
$$

Theorem 2 and the procedure described in the previous section establish the existence of tensors $C_{2}$ and $C_{1}$ such that, at every regular point $x$ of a face with normal $n$ and every regular point $y$ of an edge $\mathcal{L}_{j}$,

$$
\begin{aligned}
& \mathrm{F}_{1}^{2}(x, n)=\left(C_{2} \mid n^{\otimes 2}\right) n, \quad \mathrm{~F}_{0}^{1}\left(y, f_{j}\right)=\left(C_{2} \mid \sum_{k \in\left[\mathcal{L}_{j}\right]} v_{k}^{j} \otimes n_{k}\right), \\
& \mathrm{F}_{0}^{2}(x, n)=C_{1} \cdot n-\operatorname{div}_{\partial_{2} D}\left(\left(C_{2} \cdot n\right) \cdot \Pi_{\partial_{2} D}\right) .
\end{aligned}
$$

We recover here the expressions stated in $[24,25,31,46]$ or [12].

\subsection{Third Gradient Theory}

Let us now consider the case $N=3$ of third gradient models. The stress state reads

$$
\begin{aligned}
\mathfrak{S}(D, U)= & \int_{\partial_{2} D} \mathrm{~F}_{0}^{2}\left|U+\mathrm{F}_{1}^{2}\right| \nabla U+\mathrm{F}_{2}^{2} \mid \nabla^{2} U \\
& +\int_{\partial_{1} D} \mathrm{~F}_{0}^{1}\left|U+\mathrm{F}_{1}^{1}\right| \nabla U+\int_{\partial_{0} D} \mathrm{~F}_{0}^{0} \mid U .
\end{aligned}
$$

We have $\mathcal{O}_{n}^{3}=n^{\otimes 5}$. Moreover definition (11) implies

$$
\left(\mathcal{P}_{\mathcal{F}_{k}}^{2}\right)_{i_{1}, \ell, i_{2}, i_{3}}=\delta_{i_{1}}^{i_{2}}\left(\Pi_{\mathcal{F}_{k}}\right)_{\ell}^{i_{3}}+\left(\Pi_{\mathcal{F}_{k}}\right)_{\ell}^{i_{2}}\left(\Lambda_{\mathcal{F}_{k}}\right)_{i_{1}}^{i_{3}}
$$

and

$$
\left(\mathcal{P}_{\mathcal{L}_{j}}^{1}\right)_{\ell_{1}, \ell_{2}}=\left(e_{j}\right)_{\ell_{1}}\left(e_{j}\right)_{\ell_{2}} .
$$

Thus,

$$
\begin{aligned}
\left(\mathcal{O}_{f_{j}}^{3}\right)_{i_{1}, i_{2}, i_{3}, i_{4}} & =\sum_{k \in\left[\mathcal{L}_{j}\right]}\left(v_{k}^{j}\right)_{\ell}\left(\mathcal{P}_{\mathcal{F}_{k}}^{2}\right)_{i_{1}, \ell, i_{2}, i_{3}}\left(n_{k}\right)_{i_{4}} \\
& =\sum_{k \in\left[\mathcal{L}_{j}\right]} \delta_{i_{1}}^{i_{2}}\left(v_{k}^{j}\right)_{i_{3}}\left(n_{k}\right)_{i_{4}}+\left(n_{k}\right)_{i_{1}}\left(v_{k}^{j}\right)_{i_{2}}\left(n_{k}\right)_{i_{3}}\left(n_{k}\right)_{i_{4}}
\end{aligned}
$$


and

$$
\begin{aligned}
\left(\mathcal{O}_{w}^{3}\right)_{i_{1}, i_{2}, i_{3}} & =\sum_{j \in[\hat{x}]}\left(e_{j}\right)_{\ell_{1}}\left(\mathcal{P}_{\mathcal{L}_{j}}^{1}\right)_{\ell_{1}, \ell_{2}}\left(\mathcal{O}_{f_{j}}^{3}\right)_{\ell_{2}, i_{1}, i_{2}, i_{3}} \\
& =\sum_{j \in[\hat{x}]}\left(e_{j}\right)_{\ell_{2}}\left(\mathcal{O}_{f_{j}}^{3}\right)_{\ell_{2}, i_{1}, i_{2}, i_{3}} \\
& =\sum_{j \in[\hat{x}]} \sum_{k \in\left[\mathcal{L}_{j}\right]}\left(e_{j}\right)_{i_{1}}\left(v_{k}^{j}\right)_{i_{2}}\left(n_{k}\right)_{i_{3}} .
\end{aligned}
$$

Theorem 2 establishes the existence of a completely symmetric tensor $C_{3}$ such that, at every regular point $x$ of a face with normal $n$, every regular point $y$ of an edge $\mathcal{L}_{j}$ with shape $f_{j}$, and every wedge point $z$ with shape $w$,

$$
\begin{aligned}
& \mathrm{F}_{2}^{2}(x, n)=\left(C_{3} \mid n^{\otimes 3}\right) n \otimes n \\
& \mathrm{~F}_{1}^{1}\left(y, f_{j}\right)=\sum_{k \in\left[\mathcal{L}_{j}\right]}\left(\left(C_{3} \mid\left(v_{k}^{j} \otimes v_{k}^{j} \otimes n_{k}\right)\right) v_{k}^{j}+2\left(C_{3} \mid\left(v_{k}^{j} \otimes n_{k} \otimes n_{k}\right)\right) n_{k}\right) \\
& \mathrm{F}_{0}^{0}(z, w)=\sum_{j \in[\hat{x}]} \sum_{k \in\left[\mathcal{L}_{j}\right]} C_{3} \mid\left(e_{j} \otimes v_{k}^{j} \otimes n_{k}\right) .
\end{aligned}
$$

Lower order terms can then be computed using the procedure described in Section 5. We have

$$
\mathcal{P}_{\mathcal{F}_{k}}^{2} \mid\left(C_{3} \cdot n\right)=\left(I d+n_{k} \otimes n_{k}\right) \cdot\left(C_{3} \cdot n\right) \cdot \Pi_{\mathcal{F}_{k}}
$$

Therefore, on each face $\mathcal{F}_{k}$, we define $\mathrm{G}_{1}^{2}$ as the surface divergence:

$$
\mathrm{G}_{1}^{2}=\operatorname{div}_{\mathcal{F}_{k}}\left(\left(I d+n_{k} \otimes n_{k}\right) \cdot\left(C_{3} \cdot n\right) \cdot \Pi_{\mathcal{F}_{k}}\right)
$$

and, on each line $\mathcal{L}_{j}, \mathrm{G}_{0}^{1}$ as the line divergence

$$
\mathrm{G}_{0}^{1}=\operatorname{div}_{\mathcal{L}_{j}}\left(\sum_{k \in\left[\mathcal{L}_{j}\right]}\left(C_{3} \mid\left(n_{k} \otimes v_{k}^{j} \otimes e_{j}\right)\right) e_{j}\right) .
$$

We then rewrite the stress state $\int_{\partial_{2} D} \mathrm{G}_{1}^{2}\left|\nabla U+\int_{\partial_{1} D} \mathrm{G}_{0}^{1}\right| U$ in the canonical form $\int_{\partial_{2} D} \tilde{\mathrm{F}}_{0}^{2}\left|U+\tilde{\mathrm{F}}_{1}^{2}\right| \nabla U+\int_{\partial_{1} D} \tilde{\mathrm{F}}_{0}^{1} \mid U$ by using the surface divergence theorem, setting on each face $\mathcal{F}_{k}$

$$
\tilde{\mathrm{F}}_{1}^{2}=\left(\mathrm{G}_{1}^{2} \cdot n_{k}\right) n_{k}, \quad \tilde{\mathrm{F}}_{0}^{2}=-\operatorname{div}_{\mathcal{F}_{k}}\left(\mathrm{G}_{1}^{2} \cdot \Pi_{\mathcal{F}_{k}}\right)
$$

and, on each line $\mathcal{L}_{j}$,

$$
\tilde{\mathrm{F}}_{0}^{1}=\mathrm{G}_{0}^{1}+\sum_{k \in\left[\mathcal{L}_{j}\right]} \mathrm{G}_{1}^{2} \cdot v_{k}^{j}
$$


As shown in Section 5, the new stress state

$$
\tilde{\mathfrak{S}}(D, U)=\int_{\partial_{2} D}\left(\mathrm{~F}_{0}^{2}+\tilde{\mathrm{F}}_{0}^{2}\right)\left|U+\left(\mathrm{F}_{1}^{2}+\tilde{\mathrm{F}}_{1}^{2}\right)\right| \nabla_{\perp} U+\int_{\partial_{1} D}\left(\mathrm{~F}_{0}^{1}+\tilde{\mathrm{F}}_{0}^{1}\right) \mid U
$$

is a quasi-balanced stress state of order two. We can apply to it the results obtained in the case $N=2$ : two tensors $C_{2}$ and $C_{1}$ represent $\mathrm{F}_{1}^{2}+\tilde{\mathrm{F}}_{1}^{2}, \mathrm{~F}_{0}^{1}+\tilde{\mathrm{F}}_{0}^{1}$ and $\mathrm{F}_{0}^{2}+\tilde{\mathrm{F}}_{0}^{2}$. In [31] Mindlin introduced the operator which, to any tensor $X$, associates $L_{\partial_{2} D}(X):=-\operatorname{div}_{\partial_{2} D}\left(X \cdot \Pi_{\partial_{2} D}\right)$. Let us write our final results using this operator:

$$
\begin{aligned}
& \mathrm{F}_{1}^{2}(x, n)=\left(\left(C_{2} \mid n^{\otimes 2}\right)+L_{\partial_{2} D}\left((I d+n \otimes n) \cdot\left(C_{3} \cdot n\right)\right) \cdot n\right) n \\
& \mathrm{~F}_{0}^{2}(x, n)=C_{1} \cdot n+L_{\partial_{2} D}\left(C_{2} \cdot n+L_{\partial_{2} D}\left((I d+n \otimes n) \cdot\left(C_{3} \cdot n\right)\right)\right) \\
& \mathrm{F}_{0}^{1}\left(x, f_{j}\right)=\left(C_{2} \mid \sum_{k \in\left[\mathcal{L}_{j}\right]} v_{k}^{j} \otimes n_{k}\right)-\operatorname{div}_{\mathcal{L}_{j}}\left(\left(C_{3} \mid \sum_{k \in\left[\mathcal{L}_{j}\right]}\left(n_{k} \otimes v_{k}^{j} \otimes e_{j}\right)\right) e_{j}\right) \\
& +\sum_{k \in\left[\mathcal{L}_{j}\right]} L_{\mathcal{F}_{k}}\left(\left(I d+n_{k} \otimes n_{k}\right) \cdot\left(C_{3} \cdot n_{k}\right)\right) \cdot v_{k}^{j}
\end{aligned}
$$

The equations labeled (18a), (18b) and (18c) by MiNDLIN [31] correspond, respectively, to our equations (30), (29), (26), while the expressions for the quantities called $F$ and $\left(N_{1}, N_{2}, T_{1}, T_{2}\right)$ and $G$ page 436 in [31] correspond, respectively, to our equations (31), (27) and (28). However, the reader should be aware that, in [31], the decomposition of the plane vector $F_{1}^{1}$ in four components $\left(N_{1}, N_{2}, T_{1}, T_{2}\right)$ is unfounded.

\section{Conclusions}

We have established representation results for contact actions using as closely as possible the way followed by Cauchy for establishing the existence of the stress tensor in the first gradient theory. Our approach is based on a quasi-balance assumption which can be easily accepted for many materials. Our results have been established by applying the quasi balance inequality to some specific test fields. It is easy to check, following the algorithm described in Section 5, that the power of contact interaction can entirely be rewritten in terms of the tensors $C_{q}$ as a volume integral. Therefore the quasi-balance inequality is actually satisfied for all test fields. This means that we have drawn all possible consequences from the quasi-balance assumption.

We hope that the results stated in this paper will be considered sufficient to give an end to the controversy about the soundness of $N$-th gradient theories. The method we have presented here is not the simplest way for establishing the relationship between hyperstress tensors and generalized contact interactions. However, as it follows Cauchy's path closely, it should persuade those who consider it as the only physically based one. 
In a previous paper [13], the same results were obtained by following the D'Alembertian approach. This approach, first conceived by Lagrange for fluids and by Piola [16] for more general continuous systems, consists in postulating the form of internal virtual power (represented by a sequence of hyperstress tensors) and in computing the contact interactions which are compatible with it. As expected, the results of both approaches are the same. A careful reader could notice a difference between the boundary operators defined in the present paper and in the reference [13]. Cumbersome tensorial computations can show that they actually give the same representation of contact interactions.

It has to be emphasized again that we are far from having treated either all possible shapes for bodies (for instance, wedges where more (or less !) than three edges concur or reentrant wedges are not treated) or all possible stress states (the presented results cannot encompass stress states for which there are stress concentrations along lower dimensional manifolds, models which are needed if one wants to model for instance a fluid containing some unknown interfaces endowed with surface tension). We believe that it is a real challenge to obtain a theory à la Cauchy describing bodies in which there are concentrations of energy on lower dimensional submanifolds.

It would also be interesting, at least from a theoretical point of view, to understand what happens in a body where the order of the stress state varies from point to point, being unbounded.

\section{Appendix: Properties of Boundary Tensors}

This appendix is devoted to the proof of two lemmas which establish that the boundary tensors are completely symmetric with respect to their $p$ last subscripts. We need first to study some properties of the tensors $\mathcal{P}_{M, q}^{p}$ and $\mathcal{P}_{M}^{p}$.

The tensors $\mathcal{P}_{M, q}^{p}$ have been defined in such a way that, for any tensor $X$ of order $p$ and for any $1 \leqq q \leqq p$,

$$
\left(\mathcal{P}_{M, q}^{p} \mid X\right) \cdot \Pi_{M}=\mathcal{P}_{M, q}^{p} \mid X
$$

Hence, by summation,

$$
\left(\mathcal{P}_{M}^{p} \mid X\right) \cdot \Pi_{M}=\mathcal{P}_{M}^{p} \mid X .
$$

Extending definition (11) to the case $p=0$ by setting

$$
\mathcal{P}_{M, 0}^{p} \mid X:=X_{\perp M},
$$

we obtain, by a simple induction argument that, for any tensor $X$ of order $p$, any completely symmetric tensor $Y$ of order $p$ and for any $0 \leqq r \leqq p$,

$$
\left(\sum_{q=0}^{r}\left(\mathcal{P}_{M, q}^{p} \mid X\right)\right) \mid Y=X_{i_{1} \ldots i_{r} j_{r+1} \ldots j_{p}} \Lambda_{i_{r+1}}^{j_{r+1}} \ldots . \Lambda_{i_{p}}^{j_{p}} Y_{i_{1} \ldots i_{p}}
$$


and, in particular, $\left(\sum_{q=0}^{p}\left(\mathcal{P}_{M, q}^{p} \mid X\right)\right)|Y=X| Y$ which can be written

$$
\left(X_{\perp M}+\left(\mathcal{P}_{M}^{p} \mid X\right)\right)|Y=X| Y .
$$

The tensor $\mathcal{P}_{M}^{*}:=\sum_{q=1}^{p} \mathcal{P}_{M, q}^{p *}$ where

$$
\left(\mathcal{P}_{M, q}^{p *}\right)_{i_{1} \cdots i_{2 p}}:=\delta_{i_{1}}^{i_{p+1}} \ldots \delta_{i_{q-1}}^{i_{p+q-1}}\left(\Pi_{M}\right)_{i_{q}}^{i_{2 p}}\left(\Lambda_{M}\right)_{i_{q+1}}^{i_{p+q+1}} \cdots\left(\Lambda_{M}\right)_{i_{p-1}}^{i_{2 p-1}}\left(\Lambda_{M}\right)_{i_{p}}^{i_{p+q}}
$$

is the adjoint of $\mathcal{P}_{M}^{p}$ : for any pair $(X, Y)$ of tensors of order $p$,

$$
\left(X \mid\left(\mathcal{P}_{M}^{p} \mid Y\right)\right)=\left(\left(\mathcal{P}_{M}^{p *} \mid X\right) \mid Y\right) .
$$

When $M$ is a surface with normal $n$, then $\Lambda_{M}=n \otimes n$ and thus, for any tensor $Y$ of order $p-1$ and any vector $v$ tangent to $M$,

$$
\mathcal{P}_{M}^{p *} \mid(Y \otimes v)=\sum_{q=1}^{p}\left(Y \mid n^{\otimes p-q}\right) \otimes v \otimes n^{\otimes p-q} .
$$

We are now in position to prove the following lemma.

Lemma 7. The tensor $\mathcal{K}_{\mathcal{L}_{j}}\left(\mathcal{O}_{f_{j}}^{p} \mid X\right)$ depends only on the completely symmetric part of $X$.

Proof. Let us start by noticing that, for any tensors $X$ and $Y$ of order respectively $p$ and $p-2$,

$$
\begin{aligned}
\left(\mathcal{O}_{f_{j}}^{p-1} \mid X\right) \mid Y & =\left(\sum_{k \in\left[\mathcal{L}_{j}\right]}\left(\mathcal{P}_{\mathcal{F}_{k}}^{p-1} \mid\left(X \cdot n_{k}\right)\right) \cdot v_{k}^{j}\right) \mid Y \\
& =\sum_{k \in\left[\mathcal{L}_{j}\right]}\left(\mathcal{P}_{\mathcal{F}_{k}}^{p-1} \mid\left(X \cdot n_{k}\right)\right) \mid\left(Y \otimes v_{k}^{j}\right) \\
& =\sum_{k \in\left[\mathcal{L}_{j}\right]}\left(X \cdot n_{k}\right) \mid\left(\mathcal{P}_{\mathcal{F}_{k}}^{(p-1) \star} \mid\left(Y \otimes v_{k}^{j}\right)\right) \\
& =X \mid\left(\sum_{k \in\left[\mathcal{L}_{j}\right]}\left(\mathcal{P}_{\mathcal{F}_{k}}^{(p-1) \star} \mid\left(Y \otimes v_{k}^{j}\right)\right) \otimes n_{k}\right) .
\end{aligned}
$$

We have to prove that, for any tensors $X$ and $Y$,

$$
\mathcal{K}_{\mathcal{L}_{j}}\left(\mathcal{O}_{f_{j}}^{p-1} \mid X\right)\left|Y=\mathcal{K}_{\mathcal{L}_{j}}\left(\mathcal{O}_{f_{j}}^{p-1} \mid \mathcal{K}(X)\right)\right| Y .
$$

As the operator $\mathcal{K}_{\mathcal{L}_{j}}$ is self adjoint, it is equivalent to prove that for any tensor $X$ and any tensor $Y$ completely symmetric and orthogonal to $\mathcal{L}_{j}$,

$$
\left(\mathcal{O}_{f_{j}}^{p-1} \mid X\right)\left|Y=\left(\mathcal{O}_{f_{j}}^{p-1} \mid \mathcal{K}(X)\right)\right| Y .
$$


Owing to our preliminary remark and using identity (34), we are reduced to proving that, for any tensor $Y$, completely symmetric and orthogonal to $\mathcal{L}_{j}$, the tensor

$$
\sum_{k \in\left[\mathcal{L}_{j}\right]}\left(\mathcal{P}_{\mathcal{F}_{k}}^{(p-1) \star} \mid\left(Y \otimes v_{k}^{j}\right)\right) \otimes n_{k}=\sum_{k \in\left[\mathcal{L}_{j}\right]}\left(\sum_{q=1}^{p-1}\left(Y \mid n_{k}^{\otimes p-q-1}\right) \otimes v_{k}^{j} \otimes n_{k}^{\otimes p-q}\right)
$$

is completely symmetric. To that end, we check the invariance of this tensor with respect to the transposition of subscripts $(i, i+1)$ for all $i \in\{1, \ldots, p-1\}$.

All terms in the sum satisfying $q>i+1$ are clearly invariant owing to the complete symmetry of $Y$. The terms for which $q<i$ are also clearly invariant owing to the complete symmetry of $n_{k}^{\otimes p-q}$. So, for any $i \in\{1, \ldots p-2\}$, the only two terms in the sum which are not clearly invariant are those for which $q=i+1$ or $q=i$. The sum of these two addends reads

$$
\left(Y \mid n_{k}^{\otimes p-i-1}\right) \otimes v_{k}^{j} \otimes n_{k}^{\otimes p-i}+\left(Y \mid n_{k}^{\otimes p-i-2}\right) \otimes v_{k}^{j} \otimes n_{k}^{\otimes p-i-1} .
$$

Using the fact that $Y$ is totally orthogonal to $\mathcal{L}_{j}$, we have the equality $\left(Y \mid n_{k}^{\otimes p-i-2}\right)$. $e_{j}=0$ so that we can decompose the second of these terms in

$$
\left.\left(Y \mid n_{k}^{\otimes p-i-1}\right) \otimes n_{k} \otimes v_{k}^{j} \otimes n_{k}^{\otimes p-i-1}+\left(Y \mid n_{k}^{\otimes p-i-2}\right) \cdot v_{k}^{j}\right) \otimes v_{k}^{j} \otimes v_{k}^{j} \otimes n_{k}^{\otimes p-i-1} .
$$

Therefore the sum (35) can be rewritten

$$
\left(Y \mid n_{k}^{\otimes p-i-2}\right) \cdot\left(n_{k} \otimes\left(v_{k}^{j} \otimes n_{k}+n_{k} \otimes v_{k}^{j}\right)+v_{k}^{j} \otimes v_{k}^{j} \otimes v_{k}^{j}\right) \otimes n_{k}^{\otimes p-i-1} .
$$

The invariance of it is now clear.

We still have to deal with the case $i=p-1$. In that case there is only one term, $Y \otimes v_{k}^{j} \otimes n_{k}$, which is not clearly invariant with respect to the transposition of subscripts $p-1$ and $p$. Indeed, it is not, and we have to remind the reader that we deal with a sum over all $k \in\left[\mathcal{L}_{j}\right]$. Actually, the sum

$$
\sum_{k \in\left[\mathcal{L}_{j}\right]}\left(Y \otimes v_{k}^{j} \otimes n_{k}\right)=Y \otimes\left(\sum_{k \in\left[\mathcal{L}_{j}\right]} v_{k}^{j} \otimes n_{k}\right)
$$

is invariant as the matrix $\sum_{k \in\left[\mathcal{L}_{j}\right]} v_{k}^{j} \otimes n_{k}$ is symmetric. Indeed, let us compute its skew-symmetric part : up to a factor 2 it reads

$$
\sum_{k \in\left[\mathcal{L}_{j}\right]}\left(v_{k}^{j} \otimes n_{k}-n_{k} \otimes v_{k}^{j}\right)=R_{e_{j}}-R_{e_{j}}=0,
$$

where $R_{e_{j}}$ means the rotation of angle $\pi / 2$ about the axis $e_{j}$ and where we have used that the two bases $\left(e_{j}, n_{k}, v_{k}^{j}\right)$ for $k \in\left[\mathcal{L}_{j}\right]$ have opposite orientations. The proof is completed as the transpositions of successive subscripts generate all permutations. 
Now let us remark that, when $Y$ is a tensor of order $p-3, e$ a unit vector tangential to a line $\mathcal{L}$ and $1 \leqq i \leqq p-3$, then the quantity $\left(\mathcal{P}_{\mathcal{L}, i}^{p-2}+\mathcal{P}_{\mathcal{L}, i+1}^{p-2}\right) \mid(Y \otimes e)$ is invariant with respect to transposition of the subscripts $i$ and $i+1$. Indeed, as the subscripts different from $i$ and $i+1$ play no role, we can assume, without loss of generality, that $p-3=i=1$. In this case, the matrix $e \otimes Y_{\perp \mathcal{L}}+Y \otimes e=$ $e \otimes Y_{\perp \mathcal{L}}+Y_{\perp \mathcal{L}} \otimes e+(Y \cdot e) \otimes e \otimes e$ is clearly symmetric.

This remark is useful to prove the following lemma.

Lemma 8. The tensor $\mathcal{K}\left(\mathcal{O}_{w}^{p} \mid X\right)$ depends only on the completely symmetric part of $X$.

Proof. The proof is similar to the proof of Lemma 7. We start by noticing that, for any tensors $X$ and $Y$ of order respectively $p$ and $p-3$,

$$
\begin{aligned}
\left(\mathcal{O}_{w}^{p} \mid X\right) \mid Y & =\left(\sum_{j \in[\hat{x}]}\left(\mathcal{P}_{\mathcal{L}_{j}}^{p-2} \mid\left(\sum_{k \in\left[\mathcal{L}_{j}\right]}\left(\left(\mathcal{P}_{\mathcal{F}_{k}}^{p-1} \mid\left(X \cdot n_{k}\right)\right) \cdot v_{k}^{j}\right)\right)\right) \cdot e_{j}\right) \mid Y \\
& =X \mid\left(\sum_{j \in[\hat{x}]} \sum_{k \in\left[\mathcal{L}_{j}\right]}\left(\mathcal{P}_{\mathcal{F}_{k}}^{(p-1) \star} \mid\left(\left(\mathcal{P}_{\mathcal{L}_{j}}^{(p-2) \star} \mid\left(Y \otimes e_{j}\right)\right) \otimes v_{k}^{j}\right)\right) \otimes n_{k}\right) .
\end{aligned}
$$

We have to prove that, for any completely symmetric tensor $Y$ of order $p-3$, the tensor

$$
Z:=\sum_{j \in[\hat{x}]} \sum_{k \in\left[\mathcal{L}_{j}\right]}\left(\mathcal{P}_{\mathcal{F}_{k}}^{(p-1) \star} \mid\left(\left(\mathcal{P}_{\mathcal{L}_{j}}^{(p-2) \star} \mid\left(Y \otimes e_{j}\right)\right) \otimes v_{k}^{j}\right)\right) \otimes n_{k}
$$

is completely symmetric. Using (34), we get

$$
\begin{aligned}
\left(\mathcal{P}_{\mathcal{F}_{k}}^{(p-1) \star} \mid\left(\left(\mathcal{P}_{\mathcal{L}_{j}}^{(p-2) \star} \mid\left(Y \otimes e_{j}\right)\right) \otimes v_{k}^{j}\right)\right) \otimes n_{k} \\
=\sum_{q=1}^{p-1}\left(\left(\mathcal{P}_{\mathcal{L}_{j}}^{(p-2) *} \mid\left(Y \otimes e_{j}\right)\right) \mid n_{k}^{\otimes p-q-1}\right) \otimes v_{k}^{j} \otimes n_{k}^{\otimes p-q} \\
=\sum_{q=1}^{p-1}\left(\sum_{r=1}^{p-2} \mathcal{P}_{\mathcal{L}_{j}, r}^{(p-2) \star}\left|\left(\left(Y \otimes e_{j}\right)\right)\right| n_{k}^{\otimes p-q-1}\right) \otimes v_{k}^{j} \otimes n_{k}^{\otimes p-q} .
\end{aligned}
$$

When $q \leqq r,\left(\mathcal{P}_{\mathcal{L}_{j}, r}^{(p-2) \star} \mid\left(Y \otimes e_{j}\right)\right) \mid n_{k}^{\otimes p-q-1}=0$, otherwise, after some computation and using the complete symmetry of $Y$,

$$
\left(\mathcal{P}_{\mathcal{L}_{j}, r}^{(p-2) \star} \mid\left(Y \otimes e_{j}\right)\right)\left|n_{k}^{\otimes p-q-1}=\mathcal{P}_{\mathcal{L}_{j}, r}^{(q-1) \star}\right|\left(\left(Y \mid n_{k}^{\otimes p-q-1} \otimes e_{j}\right)\right) .
$$

Thus we get

$$
Z=\sum_{j \in[\hat{x}]} \sum_{k \in\left[\mathcal{L}_{j}\right]} \sum_{q=1}^{p-1}\left(\sum_{r=1}^{q-1} \mathcal{P}_{\mathcal{L}_{j}, r}^{(q-1) \star} \mid\left(\left(Y \mid n_{k}^{\otimes p-q-1}\right) \otimes e_{j}\right)\right) \otimes v_{k}^{j} \otimes n_{k}^{\otimes p-q}
$$


We now check the invariance of $Z$ with respect to all transpositions of subscripts $i$ and $i+1$ for $1 \leqq i<p$. This is clear, owing to the complete symmetry of $Y$, for every term in the sum (36) satisfying $r>i+1$ or $r<i<q-1$. This is also evident if $q<i$. The remark we made previously shows that the sum of a pair of terms corresponding to $r=i$ and $r=i+1$ with the same $q>i+1$ is also invariant.

When $i<p-1$ the sum of a pair of terms corresponding to $q=i$ and $q=i+1$ with the same $r<i$ is also symmetric with respect to the considered transposition. Indeed, it reads

$$
\begin{aligned}
& \mathcal{P}_{\mathcal{L}_{j}, r}^{(i-1) \star} \mid\left(\left(Y \mid n_{k}^{\otimes p-i-1}\right) \otimes e_{j}\right) \otimes v_{k}^{j} \otimes n_{k}^{\otimes p-i} \\
& \quad+\mathcal{P}_{\mathcal{L}_{j}, r}^{i \star} \mid\left(\left(Y \mid n_{k}^{\otimes p-i-2}\right) \otimes e_{j}\right) \otimes v_{k}^{j} \otimes n_{k}^{\otimes p-i-1} .
\end{aligned}
$$

However as $r<i$ implies $\mathcal{P}_{\mathcal{L}_{j}, r}^{i \star} \mid\left(\left(Y \mid n_{k}^{\otimes p-i-2}\right) \otimes e_{j}\right) \cdot e_{j}=0$, we have

$$
\begin{aligned}
\mathcal{P}_{\mathcal{L}_{j}, r}^{i \star} \mid\left(\left(Y \mid n_{k}^{\otimes p-i-2}\right) \otimes e_{j}\right)= & \left(\mathcal{P}_{\mathcal{L}_{j}, r}^{(i-1) \star} \mid\left(\left(Y \mid n_{k}^{\otimes p-i-1}\right) \otimes e_{j}\right)\right) \otimes n_{k} \\
& +\left(\mathcal{P}_{\mathcal{L}_{j}, r}^{i \star} \mid\left(\left(Y \mid n_{k}^{\otimes p-i-2}\right) \otimes e_{j}\right) \cdot v_{k}^{j}\right) \otimes v_{k}^{j} .
\end{aligned}
$$

So the sum of a pair of terms corresponding to $q=i$ and $q=i+1$ becomes

$$
\begin{aligned}
& \mathcal{P}_{\mathcal{L}_{j}, r}^{i \star} \mid\left(\left(Y \mid n_{k}^{\otimes n-i-1}\right) \otimes e_{j}\right) \otimes\left(v_{k}^{j} \otimes n_{k}+n_{k} \otimes v_{k}^{j}\right) \otimes n_{k}^{\otimes n-i-1} \\
& \quad+\left(\mathcal{P}_{\mathcal{L}_{j}, r}^{(i-1) \star} \mid\left(\left(Y \mid n_{k}^{\otimes n-i-2}\right) \otimes e_{j}\right) \cdot v_{k}^{j}\right) \otimes v_{k}^{j} \otimes v_{k}^{j} \otimes n_{k}^{\otimes n-i-1},
\end{aligned}
$$

which is now clearly invariant under the considered transposition.

From now on we must consider the whole sum defining $Z$. Indeed, we are left with some terms whose symmetry cannot be established for a fixed pair of subscripts $(j, k)$.

When $i=p-1$ the terms corresponding to $q=i$ and $r<i$ can be treated for a fixed $j$. Indeed, we have already noticed in the proof of Lemma 7 that the matrix $\sum_{k \in\left[\mathcal{L}_{j}\right]} v_{k}^{j} \otimes n_{k}$ is symmetric. Thus each sum

$$
\sum_{k \in\left[\mathcal{L}_{j}\right]}\left(\mathcal{P}_{\mathcal{L}_{j}, r}^{(p-2) \star} \mid\left(Y \otimes e_{j}\right)\right) \otimes v_{k}^{j} \otimes n_{k}
$$

is invariant with respect to the considered transposition.

The only remaining term corresponds to $r=i, q=i+1$. It can be treated for a fixed $k$. Indeed, consider the two edges (denoted $j \in[\hat{x}, k]$ ) which concur in the wedge $[\hat{x}]$ and border the face $\mathcal{F}_{k}$. We have 


$$
\begin{aligned}
\sum_{j \in[\hat{x}, k]}\left(\left(\mathcal{P}_{\mathcal{L}_{j}, i}^{(p-2) \star} \mid\left(Y \otimes e_{j}\right)\right) \mid n_{k}^{\otimes p-i-2}\right) \otimes v_{k}^{j} \otimes n_{k}^{\otimes p-i-1} \\
=\sum_{j \in[\hat{x}, k]}\left(Y \mid n_{k}^{\otimes p-i-2} \otimes e_{j} \otimes v_{k}^{j} \otimes n_{k}^{\otimes p-i-1}\right) \\
=Y \mid n_{k}^{\otimes p-i-2} \otimes\left(\sum_{j \in[\hat{x}, k]} e_{j} \otimes v_{k}^{j}\right) \otimes n_{k}^{\otimes p-i-1},
\end{aligned}
$$

which is invariant with respect to the considered transposition, as the matrix $\sum_{j \in[\hat{x}, k]} e_{j} \otimes v_{k}^{j}$ is symmetric (it skew symmetric part is $R_{n_{k}}-R_{n_{k}}=0$ ).

The proof is completed as we have explored all of the terms in the sum (36).

\section{References}

1. Aifantis, E.C.: On the role of gradients in the localization of deformation and fracture. Int. J. Eng. Sci. 30(10), 1279-1299 (1992)

2. Aifantis, E.C.: Strain gradient interpretation of size effects. Int. J. Fract. 95(1-4), 299-314 (1999)

3. Alibert J.J., Seppecher P., Dell'Isola F.: Truss modular beams with deformation energy depending on higher displacement gradients. Math. Mech. Solids 8, 51-73 (2003)

4. Altenbach, H., Eremeyev, V.A., Lebedev, L.P.: On the existence of solution in the linear elasticity with surface stresses. ZAMM J. Appl. Math. Mechan./Zeitschrift fr Angewandte Mathematik und Mechanik., 90(3), 231-240 (2010)

5. Auffray, N., dell'Isola, F., Eremeyev, V.A., Madeo, A., Rosi, G.: Analytical continuum mechanics à la Hamilton-Piola: least action principle for second gradient continua and capillary fluids. Math. Mech. Solids. 20(4), 375-417 (2015)

6. Casal, P.: La théorie du second gradient et la capillarité. C.R. Acad. Sci. Paris. 274, 1571-1574 (1972)

7. Cauchy, A.L.: De la pression ou tension dans un corps solide. Ex. de Math. 2, 4256 (available in Gallica.bnf.fr) (1827)

8. Céline, C., Boutin, C., Hans, C.: Wave propagation and non-local effects in periodic frame materials: generalized continuum mechanics. Math. Mech. Solids (2013)

9. Chesnais, C., Boutin, C., Hans, S.: Effects of the local resonance on the wave propagation in periodic frame structures: generalized newtonian mechanics. J. Acoust. Soc. Am. 132(4), Pt. 2 (2012)

10. Coleman, B.D., Noll, W.: The thermodynamics of elastic materials with heat conduction and viscosity. Arch. Ration. Mechan. Anal. 13(1),167-178 (1963)

11. DEll'Isola, F., SEPPecher, P.: The relationship between edge contact forces, double forces and interstitial working allowed by the principle of virtual power. Comptes Rendus de l'Academie de Sciences - Serie IIb: Mécanique, Physique, Chimie, Astronomie, vol. 321, 303-308 (1995)

12. DEll'isola, F., SePPECHER, P.: Edge contact forces and quasi-balanced power. Meccanica 32(1), 33-52 (1997)

13. Dell'Isola, F., SePpecher, P., Madeo, A.: How contact interactions may depend on the shape of Cauchy cuts in N-th gradient continua: approach à la D'Alembert. Zeitschrift für Angewandte Mathematik und Physik (ZAMP), 63(6), 1119-1141 (2012)

14. DELl'Isola, F., SePPECHER, P.: Hypertractions and hyperstresses convey the same mechanical information Continuum Mech. Thermodyn.2010 22: 163176 by Prof. Podio Guidugli and Prof. Vianello and some related papers on higher gradient theories. Contin. Mech. Thermodyn. 23(5), 473-478 (2011) 
15. Dell'Isola, F., Andreaus, U., Placidi, L.: A still topical contribution of Gabrio Piola to Continuum Mechanics: the creation of peri-dynamics, non-local and higher gradient continuum mechanics. In: The complete works of Gabrio Piola: Volume I, Advanced Structured Materials, vol. 38. Springer, Switzerland (2014)

16. Dell'Isola, F., Maier, G., Perego, U., Andreaus, U., Esposito, R., Forest, S.: The complete works of Gabrio Piola: Volume I. Advan. Struct. Mater. 38 (2014)

17. Del Piero, G.: Non-classical continua, pseudobalance, and the law of action and reaction. Math. Mech. Complex. Syst. 2 (1), 7110 (2014)

18. Dunn, J.E., Serrin, J.: On the thermomechanics of interstitial working. Arch. Rational Mech. Anal. 88(2), 95-133 (1985)

19. ERINGEN A.C.: Microcontinuum Field Theories. Springer-Verlag, New York (2001)

20. Forest, S., Cordero, N.M., Busso, E.P.: First vs. second gradient of strain theory for capillarity effects in an elastic fluid at small length scales. Comput. Mater. Sci. 50(4), 1299-1304 (2011)

21. FosDick, R.: Observations concerning virtual power, Math. Mech. Solids, 16, 573-585 (2011)

22. Fried, E., Gurtin, M.E. : Tractions balanced and boundary conditions for non simple materials with application to liquid flow at small length scale, Arch. Rat. Mech. Anal., 182(3), 513-554 (2006)

23. Garajeu, M., Gouin, H., Saccomandi, G.: Scaling Navier-Stokes equation in nanotubes. Phys. Fluids 25(8), 082003 (2013)

24. Germain, P.: La mthode des puissances virtuelles en mcanique des milieux continus. J. Mcanique, 12, 236-274 (1973)

25. Germain, P.: The method of virtual power in continuum mechanics. Part 2: Microstructure. SIAM J. Appl. Math. 25(3), 556-575 (1973)

26. GiUsteri, G.G.: The multiple nature of concentrated interactions in second-gradient dissipative liquids. Zeitschrift fr angewandte Mathematik und Physik, 64(2), 371-380 (2013)

27. GurTin, M.E.: Thermodynamics and the possibility of spatial interaction in elastic materials. Arch. Ration. Mechan. Anal. 195, 339-352 (1965)

28. Mindlin, R.D.: Influence of couple-stresses on stress concentrations. Exp. Mech. 36, 756757 (1962)

29. Mindlin, R.D., Tiersten, H.F.: Effects of couple-stresses in linear elasticity. Arch. Rational Mech. Anal. 11, 415-448 (1962)

30. Mindlin, R.D.: Complex representation of displacements and stresses in plane strain with couple- stresses. 1965 Appl. Theory of Functions in Continuum Mechanics (Proc. Internat. Sympos ., Tbil-isi), Mechanics of Solids (Russian), vol. I, pp. 256-259 Izdat. Nauka, Moscow (1963)

31. Mindlin, R.D.: Second gradient of strain and surface tension in linear elasticity. Int. $J$. Solids Struct. 1(4), 417-438 (1965)

32. Müller, I.: Thermodynamics of mixtures and phase field theory. Int. J. Solids Struct. 38(6), 1105-1113 (2001)

33. Neff, P., Chemiki, K., Alber, H.D.: Notes on strain gradient plasticity: finite strain covariant modelling and global existence in the infinitesimal rate-independent case. Math. Models Methods Appl. Sci. 19(02), 307-346 (2009)

34. Noll, W.: The foundations of classical mechanics in the light of recent advances in continuum mechanics, In: The Axiomatic Method, with Special Reference to Geometry and Physics, pp 266-281. North-Holland, Amsterdam (1959)

35. Noll, W., Virga, E.: On edge interactions and surface tension. Arch. Ration. Mech. Anal. 111, 1-31 (1990)

36. Pideri, C., Seppecher, P.: A second gradient material resulting from the homogenization of an heterogeneous linear elastic medium. Contin. Mech. Thermodyn. 9(5), 241-257 (1997)

37. PIola, G.: Sull'applicazione de' principj della meccanica analitica del Lagrange ai principali problemi. Memoria di Gabrio Piola presentata al concorso del premio e coronata 
dall'I.R. Istituto di Scienze, ecc. nella solennita del giomo 4 ottobre 1824, Milano, Imp. Regia stamperia (1825)

38. Piola, G.: Memoria intorno alle equazioni fondamentali del movimento di corpi qual sivogliono considerati secondo la naturale loro forma e costituzione, Modena, Tipi del R.D. Camera (1846)

39. Polizzotto, C., Borino, G.: A thermodynamics-based formulation of gradientdependent plasticity. Eur. J. Mech. A/Solids 17(5) 741-761 (1998)

40. Schwartz, L.: Theorie des Distributions, Hermann, Paris 1973

41. Sciarra, G., Dell'Isola, F, Coussy, O.: Second gradient poromechanics. Int. J. Solids Struct. 44(20), 6607-6629 (2007)

42. Segev, R., De Botton, G.: On the consistency conditions for force systems. International Journal of Non-Linear Mechanics, Elsevier, 26(1), 47-59 (1991)

43. Seppecher, P.: Moving contact lines in the Cahn-Hilliard theory. Int. J. Eng. Sci. 34(9), 977-992 (1996)

44. Seppecher, P., Alibert, J.J., Dell'Isola, F.: Linear elastic trusses leading to continua with exotic mechanical. J. Phys. Conf. Ser. 319(1), 012018 (2011)

45. Tномаs, E.G.F.: A polarization identity for multilinear maps. Indag. Math. 25(3), 468 474 (2014)

46. Toupin, R.A.: Elastic materials with couple-stresses. Arch. Ration. Mech. Anal. 11, 385-414 (1962)

47. Toupin, R.A.: Theories of elasticity with couple-stress. Arch. Ration. Mech. Anal., 17, 85-112 (1964)

48. Triantafyllidis, N., Aifantis, E.C.: A gradient approach to localization of deformation. I. Hyperelastic materials. J Elast. 16(3), 225-237 (1986)

49. Truesdell, C., Noll, W.: The Non-linear Field Theories of Mechanics, in Fliigge's Encyclopedia of Physics, vol. III/3. Springer-Verlag, Berlin, pp. 1-662 (1965)

50. Truesdell, C.: Cauchy and the modern mechanics of continua. Revue d'histoire des sciences, $t$ 45(1), 5-24 (1992)

51. YANG, Y., Misra, A.: Micromechanics based second gradient continuum theory for shear band modeling in cohesive granular materials following damage elasticity. Int. $J$. Solids Struct. 49(18), 2500-2514 (2012)

Universita di Roma La Sapienza, via Eudossiana 18,

Rome, Italy.

e-mail: fdellisola@gmail.com

and

LGCE INSA Université deLyon, 69621 Villeurbane, France.

and

Université de Toulon, IMATH, EA 2134, CS 60584, 83041 TOULON CEDEX 9 , France. 